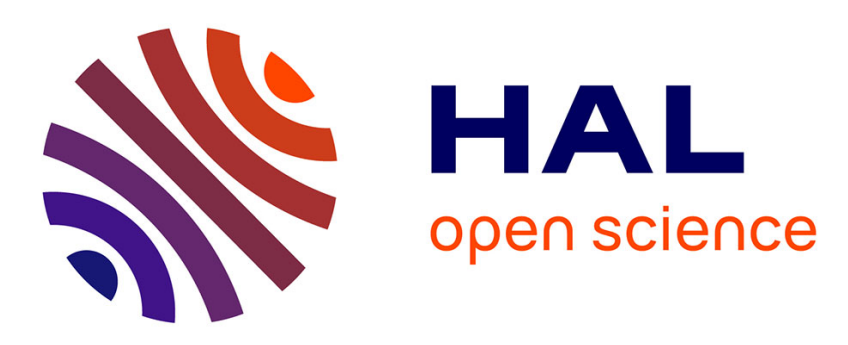

\title{
Dynamics of task-related electrophysiological networks: a benchmarking study
}

Judie Tabbal, Aya Kabbara, Mohamad Khalil, Pascal Benquet, Mahmoud Hassan

\section{- To cite this version: \\ Judie Tabbal, Aya Kabbara, Mohamad Khalil, Pascal Benquet, Mahmoud Hassan. Dynamics of task- related electrophysiological networks: a benchmarking study. NeuroImage, 2021, 231, pp.117829. 10.1016/j.neuroimage.2021.117829 . hal-03190818}

\section{HAL Id: hal-03190818 https://hal.science/hal-03190818}

Submitted on 26 May 2021

HAL is a multi-disciplinary open access archive for the deposit and dissemination of scientific research documents, whether they are published or not. The documents may come from teaching and research institutions in France or abroad, or from public or private research centers.
L'archive ouverte pluridisciplinaire HAL, est destinée au dépôt et à la diffusion de documents scientifiques de niveau recherche, publiés ou non, émanant des établissements d'enseignement et de recherche français ou étrangers, des laboratoires publics ou privés. 


\title{
Dynamics of task-related electrophysiological networks: a benchmarking study
}

\author{
Judie Tabbal a,b,*, Aya Kabbara ${ }^{\mathrm{a}}$, Mohamad Khalil ${ }^{\mathrm{b}, \mathrm{c}}$, Pascal Benquet ${ }^{\mathrm{a}}$, Mahmoud Hassan ${ }^{\mathrm{d}}$ \\ a Univ Rennes, LTSI - U1099, F-35000 Rennes, France \\ ${ }^{\mathrm{b}}$ Azm Center for Research in Biotechnology and Its Applications, EDST, Lebanese University, Beirut, Lebanon \\ ${ }^{\text {c } C R S I ~ L a b, ~ E n g i n e e r i n g ~ F a c u l t y, ~ L e b a n e s e ~ U n i v e r s i t y, ~ B e i r u t, ~ L e b a n o n ~}$ \\ ${ }^{\mathrm{d}}$ NeuroKyma, F-35000 Rennes, France
}

\section{A R T I C L E I N F O}

\section{Keywords:}

Magneto-encephalography (MEG)

Electrophysiological brain networks

Dynamic functional connectivity

Dimensionality reduction

Source separation

\begin{abstract}
A B S T R A C T
Motor, sensory and cognitive functions rely on dynamic reshaping of functional brain networks. Tracking these rapid changes is crucial to understand information processing in the brain, but challenging due to the great variety of dimensionality reduction methods used at the network-level and the limited evaluation studies. Using Magnetoencephalography (MEG) combined with Source Separation (SS) methods, we present an integrated framework to track fast dynamics of electrophysiological brain networks. We evaluate nine SS methods applied to three independent MEG databases $(\mathrm{N}=95)$ during motor and memory tasks. We report differences between these methods at the group and subject level. We seek to help researchers in choosing objectively the appropriate SS method when tracking fast reconfiguration of functional brain networks, due to its enormous benefits in cognitive and clinical neuroscience.
\end{abstract}

\section{Introduction}

Evolving evidence show that motor, sensory, emotional and cognitive functions emerge from dynamic interactions between cortical and subcortical brain structures. Specific rhythms of neural networks allow synchronization and long-range communication between distant and distributed brain areas. This phenomena was shown crucial during visual (Bola and Sabel, 2015; Hassan et al., 2015; Mheich et al., 2018), auditory (Fontolan et al., 2014), sensorimotor (Pomper et al., 2015; Wilkins and Yao, 2020) and cognitive (Negrón-Oyarzo et al., 2018; Rouhinen et al., 2020) tasks. This brain communication is very transient and there is a dynamic reorganization of functional brain networks during behavioral tasks, even at sub-second time scale (Vidaurre et al., $2018 b)$. Therefore, the analysis of whole-brain dynamic functional connectivity (dFC) has become a burgeoning field of research in cognitive neuroscience (Bassett and Sporns, 2017; Bullmore and Sporns, 2009; Iraji et al., 2020; Kabbara et al., 2020). In this regard, Magneto/Electroencephalography (MEG/EEG) provides a unique direct and noninvasive access to the electrophysiological activity of the whole brain, at the millisecond scale. Benefiting from the excellent time resolution of the MEG/EEG ( $\sim$ millisecond), current methods allow of estimating sub-second time-varying functional brain networks in the cortical space through sensor-level signals (Hassan et al., 2014; Hassan and
Wendling, 2018). The key challenge here is how to characterize and quantify these rapidly changing networks.

In this context, several frameworks have been used to explicitly model/capture dynamics over time such as Hidden Markov Model (HMM) (Baker et al., 2014; Vidaurre et al., 2018a, 2018b, 2016), Autoregressive model (AR) (Casorso et al., 2019) and General Linear Model (GLM) (Friston, 1994). For example, HMM describes the brain activity as a sequence of district states; each represents a unique pattern obtained from an observation model, and a state time course indicating the points in time at which that state is active. Other approaches analyze the time varying signal using data-driven techniques, where 'brain network states' are derived directly from the data without a priori hypothesis on the fitting model. These methods have showed promising results, despite the fact that the selection of the used algorithm is largely empirical. These methods are based on two main steps: (1) sliding window approach, that forms a series of temporal networks, (2) a dimensionality reduction or clustering approach including Kmeans (Allen et al., 2014; Ciric et al., 2017; Du et al., 2016; Fong et al., 2019; Liu and Duyn, 2013; Mheich et al., 2015; O'Neill et al., 2015), component analysis such as temporal Independent Component Analysis tICA (O'Neill et al., 2017), Principal Component Analysis (PCA) (Leonardi et al., 2013) and Nonnegative Matrix Factorization (NMF) (Chai et al., 2017). Although the conceptual difference between these methods (and within each family of methods such as different ICA algorithms) is theoretically obvious (as

\footnotetext{
* Corresponding author.

E-mail address: judytabal95@gmail.com (J. Tabbal).
} 
they are based on different assumptions), the studies that investigate the differences between them remained very few. The existing comparative studies are mainly limited to confirming results of differences between two conditions (Leonardi et al., 2013) or to prove that obtained results are unaffected by the method's choice (Miller et al., 2016). However, a throughout quantitative and qualitative comparative study using both simulation-based and data-driven approaches is still missing and there is no clear consensus about the 'best' (if any) source separation or clustering method to be used to adequately tracking $\mathrm{dFC}$, which is the main objective of our study.

Here, we evaluate the performance of nine dimensionality reduction methods used to track functional connectivity states at both group and individual levels. This was done using simulations and three independent MEG datasets $(\mathrm{N}=95)$ recorded during motor and working memory tasks (see Fig. 1). The dynamic brain networks were reconstructed using MEG source connectivity method combined with a sliding window technique. The dimensionality reduction algorithms were compared in terms of their temporal and spatial accuracy. These methods include PCA, NMF, Kmeans and six various versions of ICA (Joint Approximation Diagonalization of Eigen-Matrices (JADE), INFOMAX, Second-Order Blind Identification (SOBI), fixed-point algorithm (FastICA), COM2 and Penalized Semi-Algebraic Unitary Deflation (P-SAUD). The motivation behind using several ICA subtypes is that each one has its own definition of statistical independence and several studies showed conceptual differences between them (Kachenoura et al., 2008; Sahonero-Alvarez and Calderon, 2017). We also analyzed the optimal number of subjects needed for each method to reveal significant results. This study aims at providing a framework for researchers interested in studying reconfiguration of functional brain networks during cognitive processes.

\section{Materials and methods}

\subsection{Data}

\subsubsection{Dataset1: 'self-paced button press task'}

This dataset includes 15 healthy righthanded participants (9 male and 6 female, aged $25 \pm 4$ years (mean \pm SD)). They were asked to press a button with the index finger of their non-dominant hand, once every 30 seconds, and should not count the time between presses. More details about this dataset can be found in (Kabbara et al., 2019; O'Neill et al., 2017).

\subsubsection{Dataset2: 'HCP left hand movement Task'}

61 healthy participants (28male and 33 female, aged 22-35) completed the MEG Motor task provided by the Human Connectome Project (HCP) (MEG-1 release) (Van Essen et al., 2012). The corresponding experimental protocol was adapted from Buckner and colleagues (Buckner et al., 2011; Thomas Yeo et al., 2011). It was performed in two sessions of $14 \mathrm{~min}$ each, with a small break between them. Each session consisted of 42 total blocks randomly distributed; 32 of them were partitioned into 16 hand movements blocks ( 8 right and 8 left), and 16-foot movements blocks ( 8 right and 8 left), and the remaining 10 blocks were interleaved resting/fixation blocks. Each motor effector block was preceded by a $3 \mathrm{sec}$ visual cue that prompts participants to either tap their left or right index and thumb fingers or squeeze their left or right toes. The block lasted for $12 \mathrm{sec}$ and consisted of 10 sequential movements, each initiated with $150 \mathrm{~ms}$ pacing stimuli followed by $1050 \mathrm{~ms}$ black screen for task execution. Here, for simplicity, we were interested in the trials related to the left hand moves only. MEG data was recorded at Saint Louis University at $508.6275 \mathrm{~Hz}$ sampling frequency and co-registered with the available subject specific MRI. EMG activity was also recorded from each limb.

\subsubsection{Dataset3: 'sternberg working memory task'}

19 healthy participants (10 male and 9 female, aged $25 \pm 3$ years (mean \pm SD)) performed Sternberg task, in which two example visual stimuli, mainly abstract geometric shapes, were successively presented on a screen; each for $0.6 \mathrm{sec}$ and separated by $1 \mathrm{sec}$. Then, a maintenance period of $7 \mathrm{sec}$ was left before the presentation of a third probe stimulus. Consequently, subjects were asked to press a button with their right index finger only if the probe stimulus matched either of the two example stimuli and an immediate feedback will be given to show their response correctness. 30 trials were presented separated by 30 sec of rest. In both datasets 1 and 3, MEG data were recorded using a 275-channel CTF MEG system at $600 \mathrm{~Hz}$ sampling frequency and co-registered with subject-specific MRI. Both datasets were approved by the University of Nottingham Medical School Research Ethics Committee (O'Neill et al., 2017; Vidaurre et al., 2018a).

\subsection{Methodology}

\subsubsection{Preprocessing}

Both datasets 1 and 3 were received already preprocessed as described in (O'Neill et al., 2017). Briefly, bad segments produced by muscles, eye or head movement were already visually inspected and removed. For dataset 2, we used the preprocessing pipeline offered by the HCP consortium, which includes removing bad channels, segments and bad independent components from task data. Segments were retrieved from the dataset 1 in the interval $[-15 ;+15 \mathrm{sec}]$ relative to the button press onset, and from the dataset 3 in the interval of [-16; $+28 \mathrm{sec}$ ] relative to stimulus presentation. In HCP analysis, we chose data epochs time-locked to EMG onset as we were concerned in exploring brain networks involved during movement execution. Thus, trials were segmented in $[-1.2 ;+1.2 \mathrm{sec}]$ relative to EMG onset. Then, as functional connectivity was proved to be frequency-dependent (Baker et al., 2014; Hipp et al., 2012), each dataset was preprocessed in its appropriate frequency band actively involved in the corresponding cognitive task. While beta band [13-30Hz] was used for self-paced and HCP left hand motor task, working memory data was filtered in a broader band [4-30Hz] as it is has been shown to involve multiple frequency bands, according to previous studies (Brookes et al., 2012; O'Neill et al., 2017). After these preprocessing steps, an average of 34, 150 and 29 per subject were kept from dataset 1, 2 and 3, respectively.

\subsubsection{Source reconstruction and functional connectivity}

In order to localize brain sources and reconstruct their activities, we used the Linearly Constrained Minimum Variance Beamforming (LCMV) (ROBINSON, 1999) approach on parcellated cortex using AAL atlas ( $\mathrm{N}=78$ regions of interests -ROIs- (Gong et al., 2009)) (Hillebrand et al., 2016). This was done by registering each subject's anatomical MRI to an MNI template (Smith et al., 2004) followed by an inverse registration to the anatomical subject space. Data covariance was computed within the specific frequency band used and a time window spanning the whole experiment (Brookes et al., 2008) with a regularization parameter (5\%) using Tikhonov method. The forward model was based upon a dipole approximation (Sarvas, 1987) and a multiple local sphere head model fitted to the subject-specific MRI scalp surface. Dipole orientation was determined using a non-linear search for optimum 'signal to noise ratio' (SNR) (Sekihara and Nagarajan, 2008). Following this, we estimated the functional connectivity by computing the amplitude envelope correlations (using Hilbert transformation) between all ROIs (Brookes et al., 2012; Hipp et al., 2012). In order to avoid spurious estimates of functional connectivity, we performed leakage correction on the reconstructed sources signals. We used the multivariate approach based on symmetric orthogonalisation proposed by (Brookes et al., 2012; Colclough et al., 2015) for datasets 1 and 3, while pair-wise orthogonalization (Brookes et al., 2016; Tewarie et al., 2019b) was applied to dataset 2 due to the short time period of the task. 

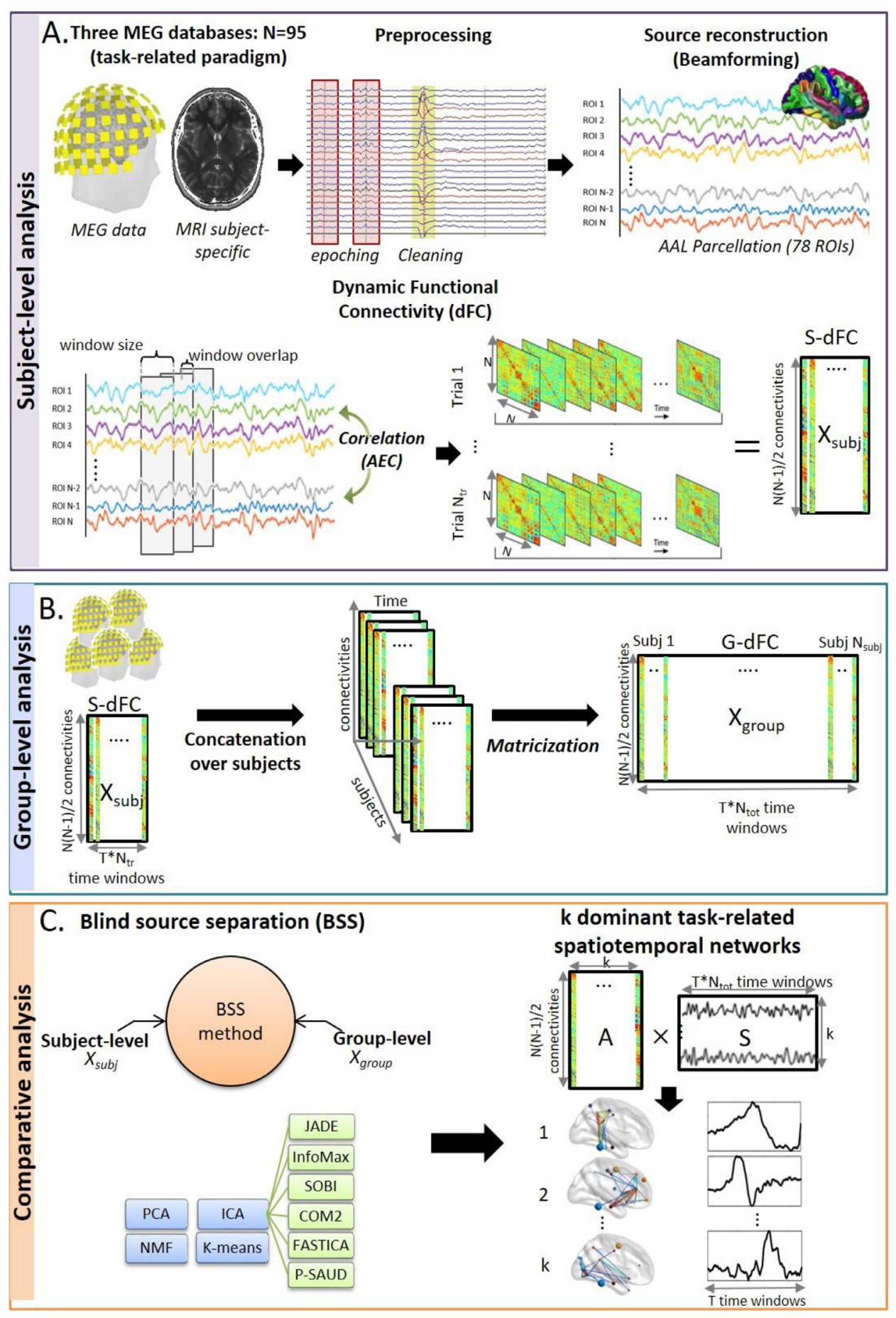

Fig. 1. Illustration of the investigation structure for each of the three task-related paradigms. A. The fundamental processing pipeline applied on each subject data from sensor-level (using non-invasive MEG technique) to cortical-level (using beamforming as the inverse problem solution) to dynamic functional connectivity computation (S-dFC) (using the sliding window approach). B. Concatenation of S-dFC of all subjects along time axis to form a group data referred to as G-dFC, C. Comparative analysis between nine different source separation (SS) methods (six variants of ICA, PCA, NMF and Kmeans) applied on both group-level $\left(\mathrm{X}_{\text {group }}\right)$ and subject-level $\left(\mathrm{X}_{\text {subj }}\right)$ data in order to derive $\mathrm{k}$ dominant task-related spatiotemporal components (the mixing matrices represent brain spatial maps while the extracted sources represent corresponding temporal weights fluctuations).

\subsubsection{Dynamic functional connectivity analysis (dFC)}

To estimate the dynamic functional brain networks, we adopted the widely used approach of sliding windows for datasets 1 and 3. To this end, a time window of length $6 \mathrm{sec}$ with $0.5 \mathrm{sec}$ was used for datasets 1 and 3 as applied by (O'Neill et al., 2017). Concerning the dataset 2 (HCP dataset), the fast time scale of the task imposes a very small time window width that may be too noisy to extract meaningful information (Liuzzi et al., 2019). Thus, we avoided to apply the sliding window approach, and used instead the high temporal resolution version of amplitude envelope correlation metric; the 'Instanteneous Amplitude Correlation' (IAC) already validated in a recent work for the same dataset
(Tewarie et al., 2019b). As a result, we obtained, for each subject trial, a 'dynamic functional connectivity (dFC)' matrix of dimension [NxNxT], where $T$ refers to the number of windows for datasets 1 and 3 , and number of total time samples for dataset $2(\mathrm{~T}=49,1221$ and 75 for datasets 1,2 and 3 respectively). Next, due to symmetry, we unfolded this matrix into a $2-\mathrm{D}[\mathrm{Nx}(\mathrm{N}-1) / 2 \times \mathrm{T}]$ matrix by removing the redundant connections in each time window. Then, the mean of each row of this matrix is subtracted from the data. Finally, all subjects' trials dFC were concatenated along the temporal dimension.We defined this matrix as a 'Group dynamic functional connectivity matrix (G-dFC)', denoted ' $\mathrm{X}$ '. Note that for dataset 2 , we averaged connectivity matrices of all trials 
relative to each subject (Zhu et al., 2020) due to memory limitation in Matlab regarding high dimensional data of the temporally concatenated 'sample-by-sample' dFC of all subject's trials.

\subsubsection{Task-related functional brain networks}

2.2.4.1. Problem statement. The resultant G-dFC matrix representing the time-varying features can be expressed as as a linear mixture of elementary brain networks that fluctuate dynamically over time. Such issue is the main concern of Source Separation (SS) approach aiming at recovering ' $k$ ' hidden sources from a set of observations with minimal priori knowledge about these sources. In this context, the SS problem can be formulated as follows:

$\mathrm{X}=\mathrm{A} \times \mathrm{S}$

Where:

- ' $\mathrm{X}$ ' is the computed G-dFC matrix of dimension [qxm]:

$\mathrm{q}=\mathrm{Nx}(\mathrm{N}-1) / 2$ with $\mathrm{N}=78$, representing connectivities between all ROIs.

$\mathrm{m}=\mathrm{TxN}_{\text {tot }}$ with $\mathrm{T}$ is the number of time windows and $\mathrm{N}_{\text {tot }}$ is the total number of trials for all subjects.

- 'A' is the mixing matrix of dimension [qxk] illustrating the contribution weights of each individual connection to the components sources, thus the spatial maps of brain networks $(\mathrm{k}<\mathrm{min}(\mathrm{q}, \mathrm{m}))$.

- ' $\mathrm{S}$ ' is the sources matrix of dimension [kxm] representing temporal sources signatures of G-dFC, collapsed across all connections.

Among existing SS algorithms, we chose nine popular/well-known methods: six different variants of temporal Independent Component Analysis (tICA), Principal Component Analysis (PCA), Non-negative Matrix Factorization (NMF) and Kmeans as a state-of-the-art clustering method. They all transform the desired matrix factorization into spatial maps and time series. However, they differ primarily in the constraints imposed on decomposed components. Below, we will give a succinct description about these methods.

2.2.4.2. Independent component analysis: 'temporal statistical independence'. ICA tends to linearly transform multivariate observations into a set of 'statistically mutually independent' latent variables under the hypothesis that these variables are as 'non-Gaussian' as possible. In our study, we examine temporal ICA (tICA) adopted by several previous studies (O'Neill et al., 2017; Yaesoubi et al., 2015) in order to obtain states that fluctuate independently in time. In this context, decomposed signals ' $\mathrm{S}$ ' consist of the ' $\mathrm{k}$ ' source time courses and the associated mixing matrix ' $A$ ' illustrates the contribution of temporally independent maps.

There are several criteria to measure independence such as minimization of mutual information and maximization of non-Gaussianity. Hence, different algorithms are proposed to perform ICA decomposition, each yielding to different ICA model with specific characteristics. Here, we evaluate tICA using six different popular and prominent methods: (1) JADE, (2) InfoMax, (3) SOBI, (4) FastICA, (5) CoM2 and (6) PSAUD. These methods are chosen in such a way to cover various statistical independence definitions, statistical order and computational process techniques. Briefly, InfoMax and FastICA are based on information theory, while all other selected methods optimize contrast functions based on cumulants of the data. Among them, SOBI uses only Second Order (SO) cumulants in contrast to others that exploit both SO and Fourth Order (FO) cumulants. In addition, FastICA and PSAUD use a deflation process for decomposition while other ICA variants jointly separate sources. Details about ICA subtypes used can be found in supplementary materials.

2.2.4.3. Principal component analysis: 'variance maximization'. PCA is a basic linear technique widely used for data dimensionality reduction. It involves a mathematical procedure that transforms a set of observations of possibly correlated variables into smaller number of orthogonal, hence linearly uncorrelated variables called principal components or 'eigenvectors. This procedure is defined in such a way that the variance or 'eigenvalues' of the data is maximized. Then, a fixed number ' $\mathrm{k}$ ' of eigenvectors and their respective eigenvalues can be chosen to obtain a consistent representation of the data. Here, we apply the Singular Value Decomposition (SVD) algorithm of PCA (Golub and Reinsch, 1970) on our predefined input matrix ' $\mathrm{X}$ '. Defining 'A' and 'S' matrices from SVD outputs is more clarified in Supplementary Materials.

2.2.4.4. Non-negative matrix factorization: 'positivity'. Nonnegative matrix factorization (NMF) is an unsupervised machine-learning technique (Lee and Seung, 1999) that imposes 'non-negativity' constraint on the decomposed factors when solving SS problem. When applied to G-dFC data ' $X$ ', NMF leads to parts-based representation that captures additive combination of basis subgraphs ' $\mathrm{A}$ ' at each time window with temporal coefficients ' $S$ ' eliminating negative signal variations. Among several existing NMF approaches, we selected Alternating Least Squares (ALS) algorithm that has previously shown good performance in fMRI context (Ding et al., 2013) with 100 times replications.

2.2.4.5. Kmeans clustering: 'sparsity'. Kmeans is one of the simplest unsupervised learning algorithms that solve the SS problem through clustering approach (Lloyd, 1982). The algorithm works iteratively to assign each point to only one of the ' $k$ ' groups based on feature similarity. Mathematical computation of Kmeans clusters is defined in Supplementary Materials. In our framework, the sparse coding adopted by Kmeans restricts a single time point to have a unique activated network state. The computed clusters ' $A$ ' represents the structure of common connectivity patterns across subjects. For a given trial, each time window is assigned with the corresponding cluster index. Then, the matrix ' $\mathrm{S}$ ' is calculated as the frequency of reoccurrence of each cluster at each time window across all trials and subjects. Here, we adapted the same procedure of Kmeans used by Allen et al. (Allen et al., 2014): L1 (Manhattan) distance is used, as it was suggested to be more effective than L2 (Euclidiean) distance for high-dimensional data (Aggarwal et al., 2001). The algorithm is replicated 100 times to increase chances of escaping local minima, and centroid positions were randomly initialized. Then, Kmeans returns the solution with the lowest 'SUMD' (within-cluster Sums of points-tocentroids Distances).

\subsubsection{Comparative analysis}

\subsubsection{MEG group-level analysis.}

2.2.5.1.1. Selection of optimal number of components $\left(N C_{\text {opt }}\right)$. In the context of dimensionality reduction methods, the choice of the optimal number of components $\left(\mathrm{NC}_{\text {opt }}\right)$ to be extracted is still a challenging issue. Here, we used the well-known approach: 'Elbow criterion' (Allen et al., 2014) for Kmeans method (with maximum number of clusters=10). For all other SS methods, we estimated $\mathrm{NC}_{\text {opt }}$ based on the goodness of fit approach (Timmerman and Kiers, 2000; Wang et al., 2018) previously used by many recent works (Tewarie et al., 2019b; Zhu et al., 2020). We performed the 'DIFFIT' method that refers to the difference in data fitting with a range of input NC varied from 2 (for motor tasks) and 4 (for working memory task) to 10 components, and selected the NC that gives the largest DIFFIT value as the $\mathrm{NC}_{\text {opt }}$. Technical details about these approaches can be found in the Supplementary Materials.

2.2.5.1.2. Selection of significant components. Among the $\mathrm{NC}_{\mathrm{opt}}$ extracted components, identifying those that reflect genuine brain activity related to the task is critical. In this paper, we followed a testing procedure adopted by (O'Neill et al., 2017) and previously described in (Hunt et al., 2012; Winkler et al., 2014) to determine significant components modulated by the tasks. The testing relies on the construction of empirical null distribution based on a 'sign flipping' permutation approach. Therefore, a component was considered significant if, at any time point, the corresponding time signal, averaged over trials, fell outside a threshold defined at 0.05 with corrections. For all datasets, 2tailed distribution was allowed, and Bonferroni corrections were applied for multiple comparisons across the NCopt components and across tem- 
poral degree of freedom. More details about 'sign-flipping' approach and threshold values setting can be found in Supplementary Materials.

2.2.5.2. MEG subject-level analysis. Besides group-level analysis, it is crucial to test the performance of each method when applied directly on individual $\mathrm{dFC}$. To this end, instead of concatenating trials from all subjects as in the final step of 'G-dFC' computation, we perform, for each subject, a dFC concatenation of all trials related only to this subject to form a subject specific dFC, denoted 'S-dFC'. Then, all selected SS methods were applied on 'S-dFC' matrix to extract subject-specific spatial and temporal signatures $(\mathrm{k}=10)$. In order to quantitatively evaluate and compare methods strength at subject-level context, we measure, for each method, both spatial and temporal similarities between each extracted S-dFC component and significant G-dFC components. These parameters are:

(1) Average Distance (AD) for 'spatial similarity':

$A D=\frac{\sum_{s} d\left(n_{s}, n_{g}\right)}{N_{s}} s \in\left[1, N_{s}\right] ; g \in\left[1, N_{G}\right]$

Where $d\left(n_{s}, n_{g}\right)$ is the Euclidian distance between the node $n_{s}$ of S-dFC network and the nearest node $n_{g}$ from the significant G-dFC network. $N_{s}$ is the total number of nodes in S-dFC network, and $N_{G}$ denotes the total number of nodes in G-dFC network. All networks were 70\% thresholded. Lower values of $\mathrm{AD}$ indicate stronger spatial similarity between S-dFC and G-dFC networks.

(2) Correlation Signals (CS) for 'Temporal Similarity':

$$
\begin{aligned}
C S(T S, T G)= & \frac{\sum_{s} \sum_{g}\left(T S_{s g}-\overline{T S}\right)\left(T G_{s g}-\overline{T G}\right)}{\sqrt{\left(\sum_{s} \sum_{g}\left(T S_{s g}-\overline{T S}\right)^{2}\right)\left(\sum_{s} \sum_{g}\left(T G_{s g}-\overline{T G}\right)^{2}\right)}} \\
& s \in\left[1, L_{s}\right] ; g \in\left[1, L_{G}\right]
\end{aligned}
$$

Where $T S$ is the temporal signal of each S-dFC component of length $L_{s}$ and $T G$ represents temporal signals of G-dFC significant component of length $L_{G}$. Higher values of CS reveal stronger temporal similarity between S-dFC and G-dFC signals.

We perform this analysis on each subject among the 15 subjects of the MEG dataset 1 (Motor task). Therefore, for each method, we counted the number of subjects that show satisfactory results performance in the context of S-dFC, based on the previously explained measures. Then, to approximate the number of subjects/trials needed for each SS method to give significant results, we follow the same procedure explained above, but instead of single subject S-dFC computation, we increased the number of concatenated subjects in $\mathrm{dFC}$ computation from $\mathrm{N}_{\text {subj }}=2$ to 14 , progressively. In order to have generalized and reliable results, we considered all possible combinations relative to each $\mathrm{N}_{\text {subj }}\left(C_{N_{\text {subj }}}^{15}\right)$, where different sets of $\mathrm{N}_{\text {subj }}$ subjects were selected among the 15 existing data subjects.

\section{Results}

In the following, we present our evaluation study on real MEG data, however our methodology was also tested on simulated data. These results can be found in the supplementary material. Briefly, the simulation-based analysis showed that all methods provide satisfactory results in terms of spatial and temporal similarity between reconstructed and simulated components with the best performance for NMF method and the worst for SOBI. All methods, except for FastICA, NMF and Kmeans, provided consistent results. PSAUD and PCA were the fastest. Results revealed that SOBI, NMF and Kmeans converge more slowly than others with the increased value of SNR. Reader can refer to supplementary material to see the detailed quantitative analysis on simulated data.

We firstly ran each algorithm at each value of NC and calculated the corresponding DIFFIT values in order to select the optimal number of components $\left(\mathrm{NC}_{\mathrm{opt}}\right)$ relative to these SS methods. Results are shown in
Fig. 2 for the three empirical datasets. Hereinafter, we set NC to the computed $\mathrm{NC}_{\text {opt }}$ value relative to each method and task.

Results of different SS methods applied on empirical data are illustrated in Figs. 3-5. In each Fig., we presented only the components that demonstrated significant task modulation based on the applied null distribution approach (described in the methods section). The networks were thresholded only for visualization purpose (70\% for dataset 1 and 3 , $85 \%$ for dataset 2). Corresponding dynamic reconfiguration of each significant network were plotted together. The temporal fluctuations represent component time signals averaged over trials and subjects.

\subsection{Self-paced button press task}

In this task, participants were asked to press a button with the index of their non-dominant hand every 30 seconds.

Based on literature findings (see table $S 1$ in supplementary materials), we were interested in quantifying SS methods ability to extract a sensorimotor network from significant components. To this end, we defined a brain network with activated AAL regions in both motor cortex (including precentral, paracentral, rolandic and supplementary motor areas) and somatosensory cortex (including postcentral, parietal and supramarginal areas) serving as a mask template for our network of interest (sensorimotor network), illustrated in Fig. 7. Then, we selected each significant network and computed the strength of each activated node in that significant network (defined as the sum of all edges weights connected to that node). The ratio of the strength of activated AAL nodes that belongs to sensorimotor mask is calculated relative to the strength of all activated nodes in that significant network. In case the ratio is greater than a certain threshold value, the network is considered as a sensorimotor network denoted as 'mot' in the Fig. 3. Otherwise, the network is denoted 'Aux' referring to auxiliary network. After many trials (threshold $=0.5,0.6,0.7$ ), threshold value was set to 0.6 as it has shown more convenient results, when visually inspecting components classification (false positive and false negative). The reader can refer to supplementary materials (Fig. S8) for more details about the computation of the ratio values for all components.

Fig. 3 shows that all SS methods were able to extract at least one significant 'Mot' network. All significant components extracted from the five ICA methods (JADE, InfoMax, FastICA, CoM2 and PSAUD), NMF and Kmeans methods were categorized as 'Mot' network due to the strong participation of sensorimotor nodes in these networks (sensorimotor strength ratio $>0.6$ ) although some of them may involve additional few connections to other regions. On the other hand, SOBI and PCA methods showed 'Aux' networks (sensorimotor strength ratio $<0.6$ ) besides 'Mot' networks, with remarkable activations in frontal regions. Temporal variation was similar for almost all significant components over all methods showing a peak value at 0sec, the button press time, with slight differences in amplitude values, indicating signal intensities relative to each component. Note that negative connectivity, referred to as blue connections in spatial networks and negative temporal values in temporal signals, represents desynchronization between brain regions. Therefore, all studied SS methods were able to extract at least one significant component that highlight strong connections between sensory and motor regions modulated significantly by the task at the exact button press instant ('Mot').

\subsection{Left-hand movement task}

This task is also motor but different than the previous one. Here the participants were asked to rapidly and successively tap their left index and thumb fingers. Similarly to the previous task, the same sensorimotor mask was applied to quantify resultant networks to discriminate 'Mot' from 'Aux' networks. The reader can refer to supplementary materials (Fig. S9) for more details about the computation of the ratio values for all components. 

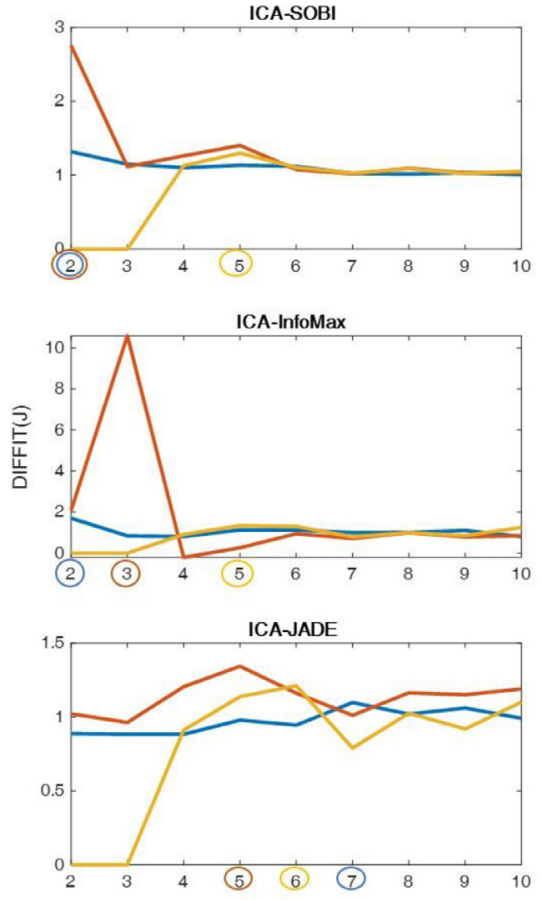
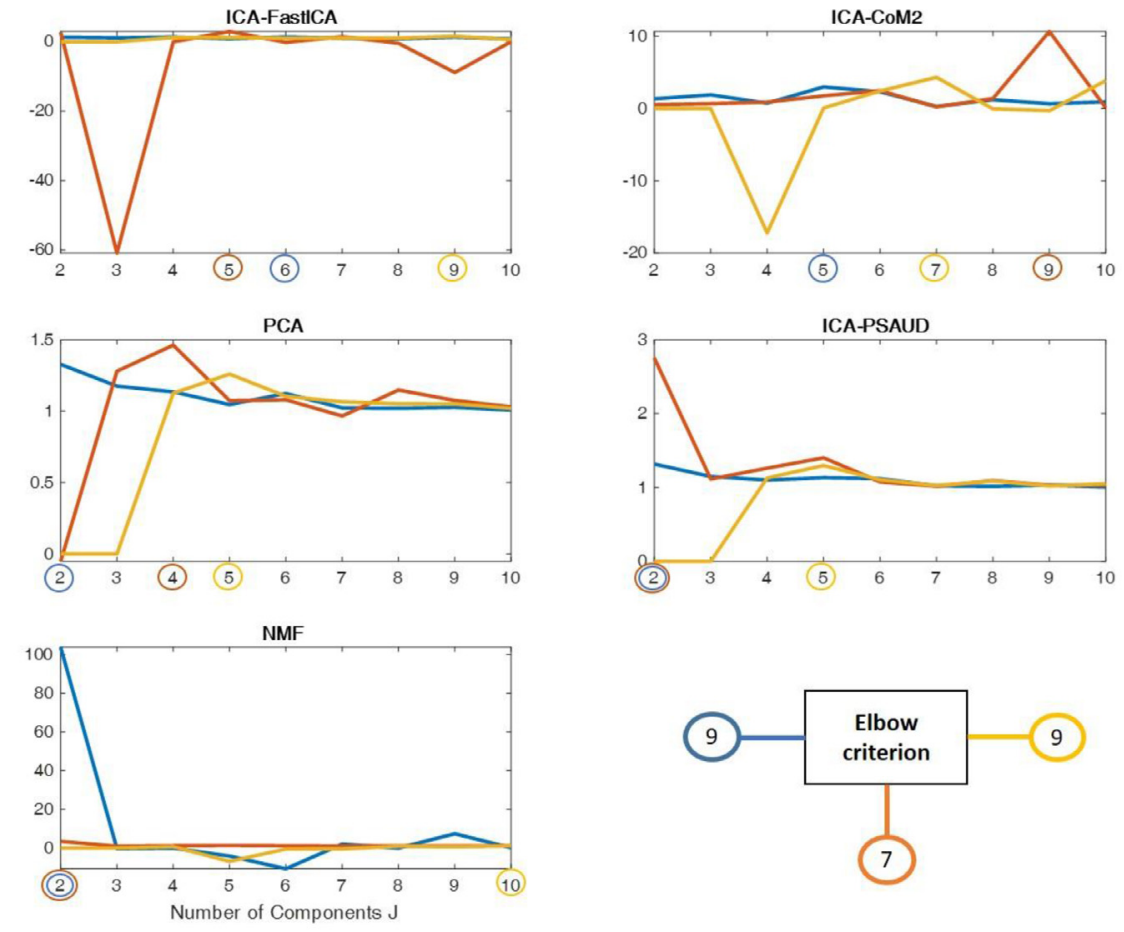

Self-paced button press task

HCP Left-Hand Movements task

Sternberg Working Memory task

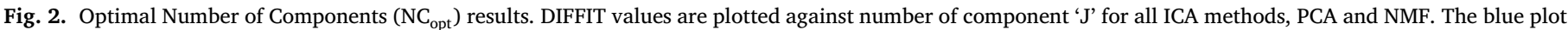

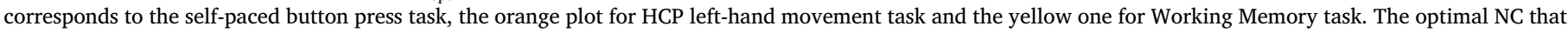

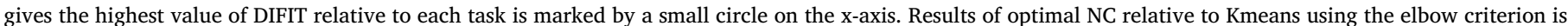
also shown. (For interpretation of the references to colour in this figure legend, the reader is referred to the web version of this article.)

Fig. 4 shows that not all SS methods were able to extract a sensorimotor 'Mot' network. For example, none of the significant components of SOBI, PSAUD and Kmeans has survived the threshold imposed for the strength of activated sensorimotor nodes (Fig. S9) and are therefore considered as 'Aux' networks as indicated in Fig. 4. In these methods, 'Aux' networks consist of either a visual network significantly modulated directly after the onset of movement in Kmeans and $0.35 \mathrm{sec}$ before onset in SOBI and PSAUD, or a network (one in SOBI and PSAUD and six in Kmeans) involving strong connections between almost all brain areas modulated at $0.2 \mathrm{sec}$ before and after onset. It should be noted that although this network shows strong activation of the right precentral and postcentral nodes, it failed to be quantified as a sensorimotor network due to the high coverage of the brain.

All remaining SS methods were able to extract one 'Mot' network among all significant components. The spatial representation of 'mot' network involves sensorimotor with some cingulate nodes from the left cortex in JADE, right cortex in InfoMax and both left and right cortices in other SS methods. These 'Mot' networks show significant drop in connectivity around $0.2 \mathrm{sec}$ following the movement onset. Significant increased modulation was also observed at $-0.2 \mathrm{sec}$ in InfoMax, FastICA, CoM2, PCA and NMF methods. Exact times of components significance are indicated with stars on Fig. 4. From this Fig., we can see that 'Aux' networks show various spatial patterns between SS methods (such as the integration of areas from visual, motor-frontal, motor-visual, temporal lobes...).

Regarding temporal evolution, the hand movement here are much more frequent than the previous task. Clearly, the fast neural activity due to the short time between successive button presses is expressed as an oscillatory behaviour of brain network activity around the zero time button press, as showed also previously (Vidaurre et al., 2018a).
This yields to the obtained temporal variation where the motor network state seems to have high connectivity before button press and begin to have a drop-in connectivity to reach its significant peak after $\sim 0.2 \mathrm{sec}$ (referred to as a desynchronization in high frequencies (Vidaurre et al., 2018a)).

\subsection{Working memory task}

This task is much more complex comparing to the other two tasks. Subjects here were asked to visualize and memorize two visual shapes and respond to a third probe stimulus by a button press (with their right index finger) in case of matching. The increased cognitive load evoked by the Sternberg task is expected to induce variations in a greater number of significant brain networks including stimulus visualisation (visual network), semantic processing and pattern recognition (semantic, language networks) and button press response (sensorimotor network).

In a similar context of previous tasks, four masks were defined here related to the most relevant working memory related networks found in literature. These masks are also illustrated in Fig. 7. The visual mask consists of the activation of primary visual cortex (occipital areas, cuneus, calcarine and lingual) and is denoted as 'Vis' in the Fig. 5. The semantic mask involves connections between bilateral temporal (including fusiform, heschl, parahippocampal) and parietal lobe (postcentral, supramarginal, angular, precuneus). The language mask (denoted 'Lang') is defined as a left lateralised network with activation of nodes from temporal, frontal and parietal regions from the left cortex. The sensorimotor mask ('Mot') is previously defined in motor tasks. Detailed ratio values of the four masks for all components can be found in details in supplementary materials (Fig. S10). In this task, by applying 


\section{Self-paced Button press}

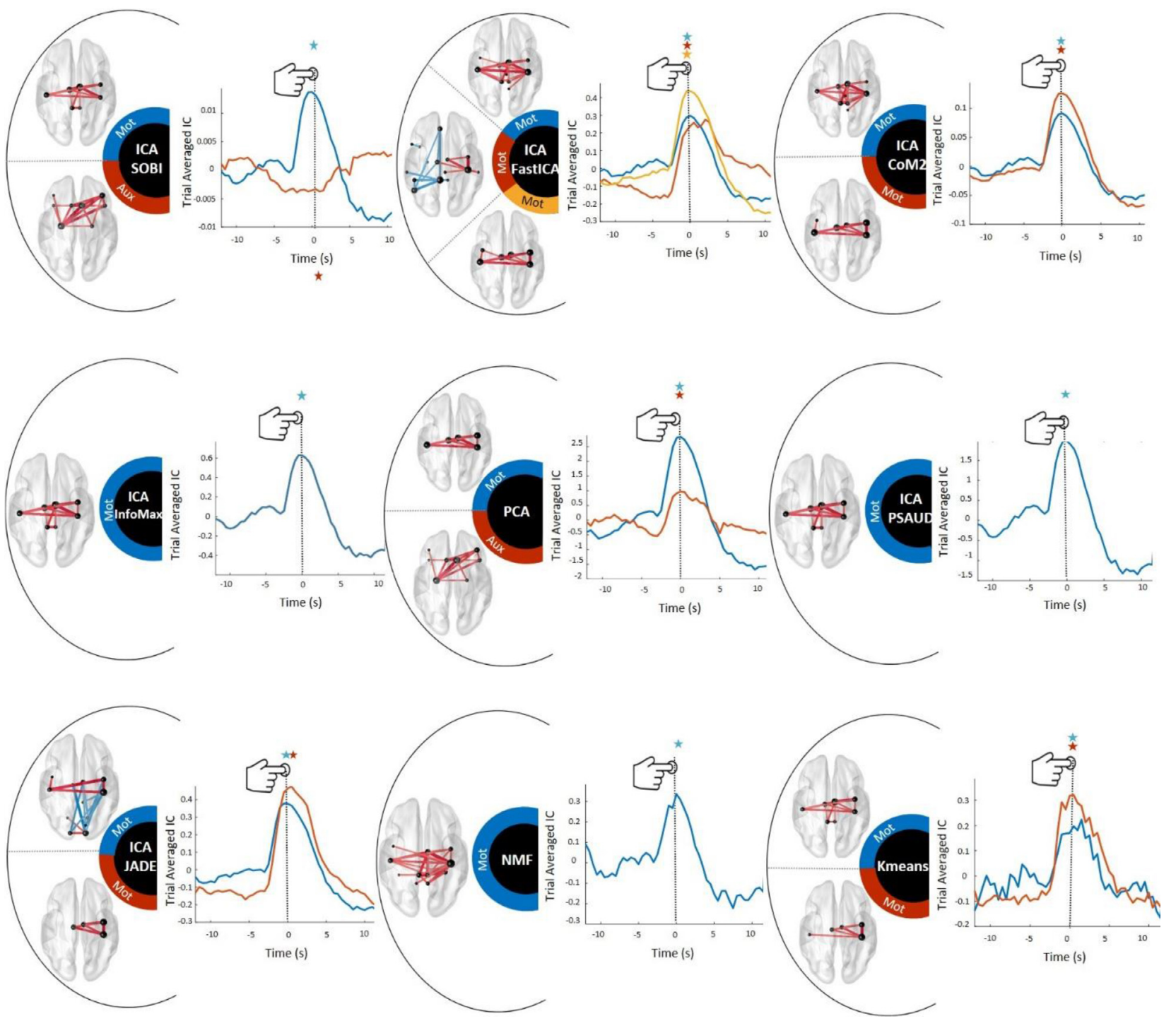

Fig. 3. Self-paced motor task results. Spatial and temporal distribution of all significant components derived from all compared SS methods applied on G-dFC in the self-paced motor task ( $\mathrm{N}=15$ subjects). All brain networks were thresholded for visualization; lines width indicates connectivity strength between regions. Red lines represent positive connectivity values while the blue ones represent negative values. Integrated AAL nodes are represented by spheres of different sizes that reveal connectivity weights (strength) between that region and the rest of brain. Corresponding temporal evolution is averaged across all trials and subjects. Time values on the $\mathrm{x}$-axis represent the position of the sliding window's center, relative to the button press at $\mathrm{t}=0 \mathrm{sec}$ (as illustrated by a vertical line). A color code is attributed for each component in space and time. For each SS method, only significant components ( $\mathrm{p}_{\text {corrected }}<0.05$ ) that appear outside the 'sign-flip' based null distribution (as described in methods sections) are shown here. All $\mathrm{NC}_{\text {opt }}$ extracted components with corresponding null distribution are shown in Supplementary Fig. S4 for an example of ICA-JADE method. Note that sensorimotor network is clearly activated at the button press instant in all SS methods. In this Fig., 'Mot' refers to sensorimotor network and 'Aux' refers to all others 'non-sensorimotor' networks. An interactive version of ICA-JADE results can be found on our github https://github.com/judytabbal/dynbrainSS.git using rotatable MATLAB figures. (For interpretation of the references to colour in this figure legend, the reader is referred to the web version of this article.)

simultaneously four masks on the same network component, there is a possibility that the ratio strength of more than one mask survives the threshold (0.6). In this case, the network belongs to the mask that gives the highest ratio strength value. In case of equality between two masks, we consider that the network belongs to both masks.

Fig. 5 illustrates the spatial distribution of all significant components for all SS methods, and temporal variation for three of these methods (JADE, NMF and Kmeans) for visualisation clarity. The temporal evolution of other SS methods can be found in Supplementary Fig. S7.
Starting from $\mathrm{t}=0 \mathrm{sec}$, two visual stimulus (shapes) were presented successively, each for $0.6 \mathrm{sec}$. During this period, all methods, except for PCA, were able to extract one or more significant 'Vis' network. We can notice few additional connections from occipital to parietal or temporal regions in this network. Time variation of this network shows significant peak during the first two seconds period.

Following stimulus presentation, subjects should retain the observed shapes in working memory. During this period, known as the maintenance phase, all methods, except Kmeans, show significant decreased modulation at [4-6sec] of at least one 'Sem' network. The breakdown in 


\section{HCP Left Hand Movement}
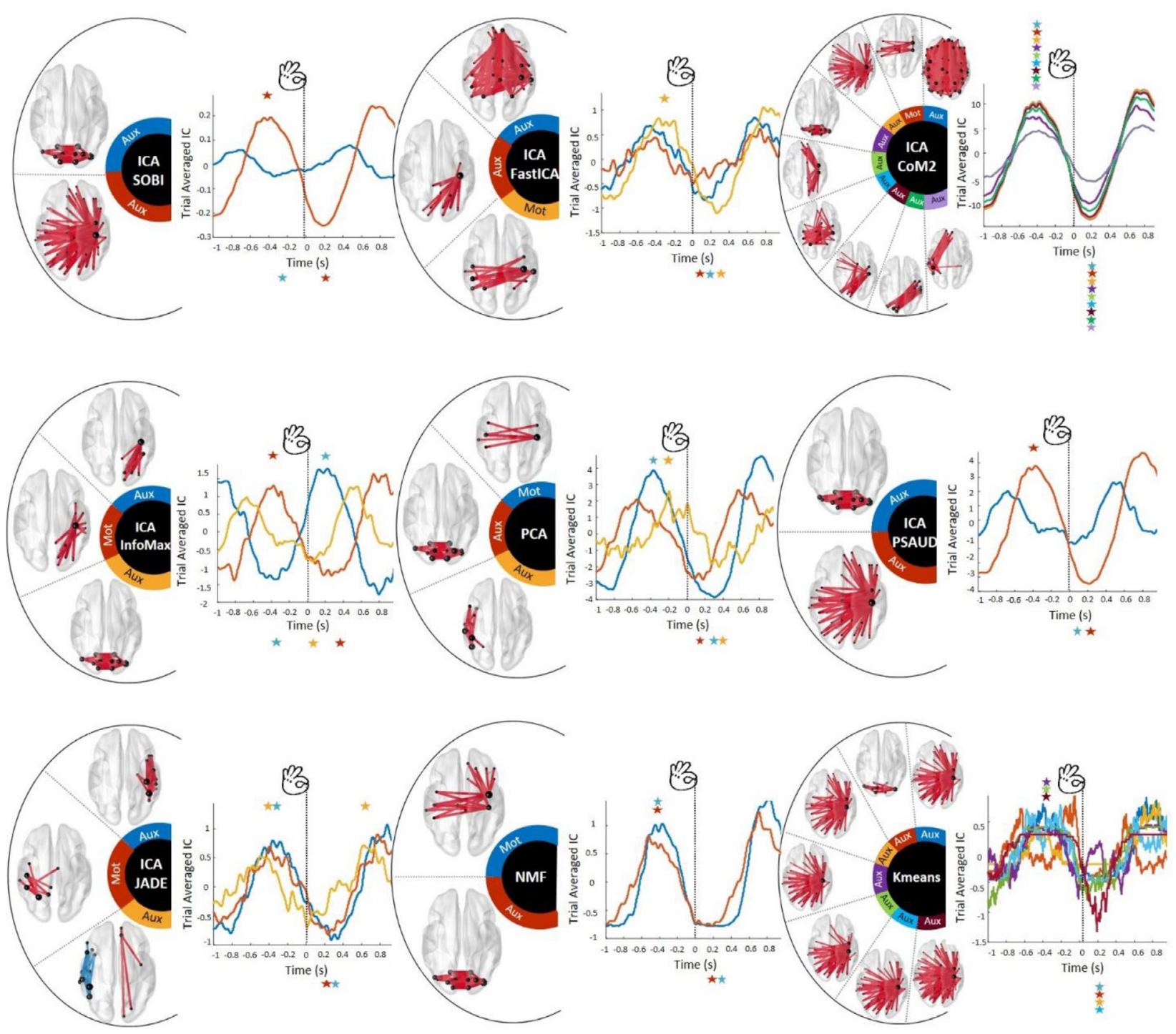

Fig. 4. HCP left hand movements task results. Spatial and temporal distribution of all significant components derived from all compared SS methods applied on $\mathrm{G}$-dFC in the left-hand motor task (N2=61subjects). All brain networks are thresholded for visualization. Time values on the $\mathrm{x}$-axis represent the position of the sliding window's center, relative to the button press at $\mathrm{t}=0 \mathrm{sec}$ (as illustrated by a vertical line). A color code is also attributed for each component in space and time. For each SS method, only significant components (pcorrected<0.05) that appear outside the null distribution are shown here. Exact times of significance relative to each component are indicated with stars., revealing an oscillatory temporal activation of motor component for all SS methods. All $\mathrm{NC}_{\text {opt }}$ extracted components with corresponding null distribution are shown in Supplementary Fig. S5 for an example of ICA-JADE method. In this Fig., 'Mot' refers to sensorimotor network and 'Aux' refers to all others 'non-sensorimotor' networks. An interactive version of ICA-JADE results can be found on our github https://github.com/judytabbal/dynbrainSS.git using rotatable MATLAB Figs.. Reproducing these results is also possible/available using the MATLAB interface on github.

this network's connectivity was previously demonstrated (O'Neill et al., 2017). During the same period, we can notice a drop-in connectivity in 'Sens' network revealed only by NMF method.

At $[10-12 \mathrm{sec}]$ period, many networks seem to be significantly modulated among all methods: (1) 'Vis' is re-activated at the probe stimulus presentation in all methods except for PSAUD, (2) 'Sens' shows significant increase with FastICA method. This network becomes most strongly connected around the time button press response, (3) 'Lang' is commonly derived by JADE, InfoMax, and PCA, and exhibits an increased connectivity peaking during probe presentation. We can notice that this network is also significantly decreased in CoM2, PSAUD and Kmeans around 5sec. (4) Three methods (JADE, InfoMax and PCA) also showed significant increased modulation of 'Sem' network. (5) A network that belongs equally to both 'Sem' and 'Sens' masks (denoted as 'Sem+Sens' network) is strongly activated during this period in CoM2 and PSAUD. Note that these two masks have common brain regions mainly in parietal lobe responsible for sensory processing which is coherent with the task evolution. This 'Sem+Sens' network is also modulated in PCA method. Besides these components, few 'Aux' networks were also considered significant as shown in Fig. 5.

In summary, the three SS methods (CoM2,PSAUD and PCA) succeeded to derive all expected components with their appropriate temporal significant modulation. JADE and InfoMax were able to extract visual, semantic and language but not the sensorimotor network. FastICA and NMF missed the language component. However, SOBI was unable to show both sensorimotor and language networks and Kmeans failed to extract semantic and sensorimotor components. 


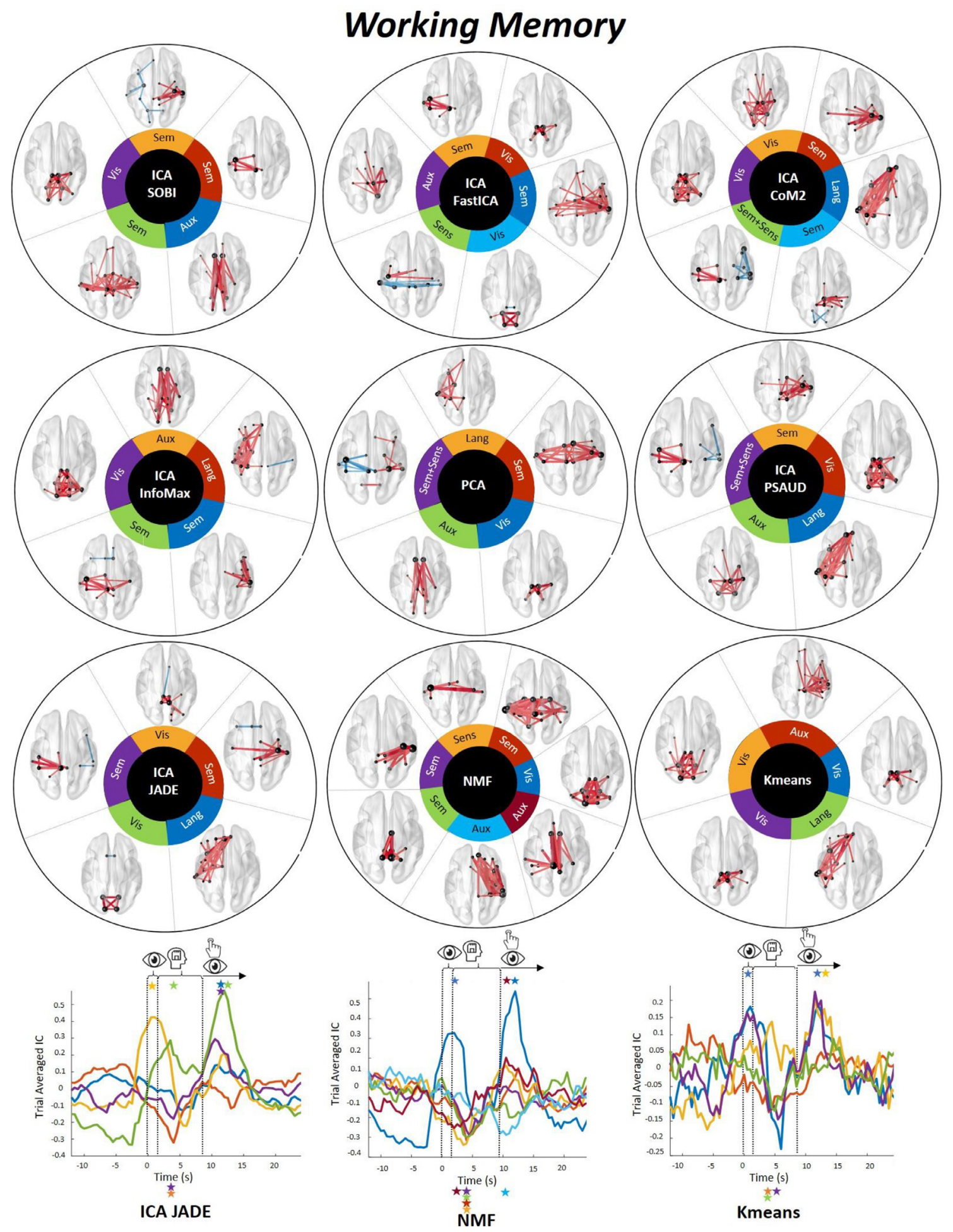

Fig. 5. Sternberg working memory task results. Spatial and temporal distribution of all significant components derived from all compared SS methods applied on $\mathrm{G}$-dFC in the working memory task $\left(\mathrm{N}_{3}=19\right.$ subjects). All brain networks are thresholded for visualization. Time values on the $\mathrm{x}$-axis represent the position of the sliding window's center, relative to the first visual stimulus presentation at $\mathrm{t}=0 \mathrm{sec}$ (as illustrated by a vertical line). The first two vertical lines illustrate the instant of successive visual examples presentation at $\mathrm{t}=0 \mathrm{~s}$ and $1.6 \mathrm{sec}$ and the third vertical line at $\mathrm{t} \sim 9 \mathrm{sec}$ separates between the maintenance period that lasts for $7 \mathrm{sec}$ and the probe presentation followed by a possible button press and feedback. A color code is attributed for each component in space and time. For each SS method, only significant components $\left(\mathrm{p}_{\text {corrected }}<0.05\right)$ that appear outside the null distribution are shown here. Exact times of significance relative to each component are indicated with stars. Temporal variation of only JADE, NMF and Kmeans is illustrated, whereas the rest are shown in Supplementary Fig. S7. All NC opt $_{\text {extracted }}$ components with corresponding null distribution are shown in Supplementary Fig. S6 for an example of ICA-JADE method. Note that in this task, much larger variety of significant networks are extracted among SS methods, including visual, sensorimotor, language, semantic, and other networks at different temporal activation. In this Fig., 'Vis' refers to Visual network, 'Sem' to Semantic, 'Sens' to Sensorimotor, 'Lang' to Language and'Aux' to other networks. An interactive version of ICA-JADE results can be found on our github https://github.com/judytabbal/dynbrainSS.git using rotatable MATLAB figures. 


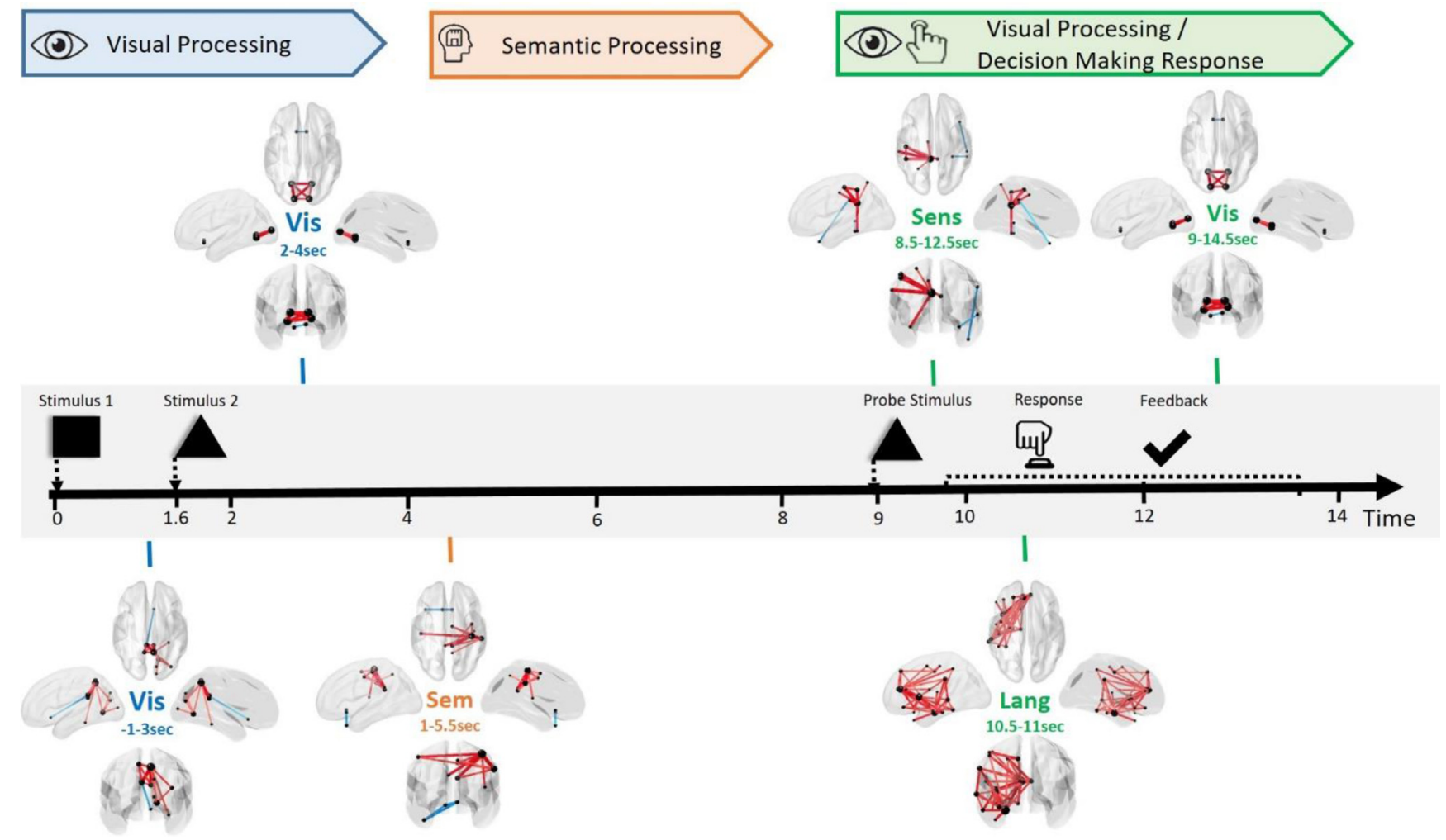

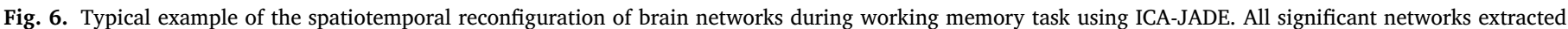

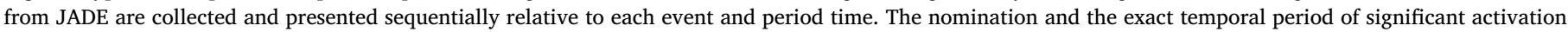

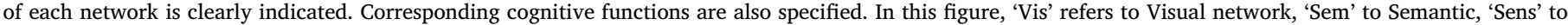
Sensorimotor, 'Lang' to Language and 'Aux' to other networks.

For the three tasks, spatial and temporal distribution of the $\mathrm{NC}_{\mathrm{opt}}$ components derived from JADE method, with corresponding null distribution, can be found in Supplementary Figs. S3, S4, S5. For further clarity in visualisation and interpretation, we illustrated, in Fig. 6, the spatiotemporal reconfiguration of the functional brain networks as obtained by ICA-JADE.

Furthermore, we discriminated different SS methods performance in terms of the activation of relevant brain networks in each task. For example, in motor tasks, we calculated the 'Mot' network occupancy percentage defined as the number of 'Mot' networks (quantitatively defined by the mask as explained above) divided by the total number of significant components found in the corresponding SS method. Similarly, for working memory task, the occupancy percentage of visual, semantic, language, sensorimotor and auxiliary networks were evaluated for each method. Results are shown in Fig. 7 with the spatial representation of the corresponding relevant brain regions. Therefore, Fig. 7 resumes the overall performance of each SS method showing variability in the methods' ability to directly extract the appropriate task-related components.

\subsection{Performance of each SS methods at subject-level}

Here our objective is to evaluate the performance of the methods at the subject-level. We test i) the capacity of each method to extract significant components related to the task: to do so, we computed the correlation between the components obtained by each method on each subject with the significant network obtained at the group level and ii) the number of subjects needed for each method to detect 'expected' networks: here we tested the overall performance of each method by increasing the number of subjects, going from 1 to 15 as we performed subject-level analysis on the self-paced data. Fig. 8.A summarizes the subject-level analysis scenario. For each method, we chose one of the significant motor components derived from the decomposition of the group-level ( $\mathrm{N}=15$ subjects), mostly the one showing little intervention from regions other than sensorimotor ('Mot') and having high temporal coefficients amplitude (supposed to be the best for each method). This component illustrates a 'group' motor network with temporal modulation at the button press time. It will, eventually, serve as a 'mas' component for subject-level analysis, as we are concerned in motor component extraction.

For each SS method, $\mathrm{NC}=10$ components were derived from each subject data. Then, Average Distance (AD) and Correlation Signals (CS) between each of these components and the 'group' motor component relative to the SS method were computed in order to quantify the ability of the method to extract, from a single subject, a task-related component in space (motor network) and time (temporal modulation at button press time) respectively. Following this, only one of these 10 components is selected for results calculation. This selection is based on two conditions criteria on $\mathrm{AD}$ and $\mathrm{CS}$ values. In the case where $\mathrm{AD}$ component is less than a threshold (set as the average of $\mathrm{AD}$ values of all components for all subjects and SS methods), and CS is higher than 0.7 (chosen as a trade-off between moderate and high correlation), then the component is considered to be a motor component. By setting these thresholds, we considered the existence of inter-subject differences, thus, allowing subjects to have different but near spatial distribution of motor network. Therefore, if at least one of the extracted components pass these conditions, corresponding $\mathrm{AD}$ and $\mathrm{CS}$ values are denoted and the number of subjects that give similar results to group-level is raised by 1 . Otherwise, we selected component $i$ as the nearest component to the group-level result, with a compromise between spatial and temporal similarities.

A typical example is illustrated in Fig. 8.A showing that PCA decomposition was able to extract a motor component from subject 2 (component 5), whereas no motor component was derived from subject 14 , $\mathrm{AD}$ and CS values of component 7 were denoted in this case. Spatial and 


\section{A. Self-paced Motor Task}
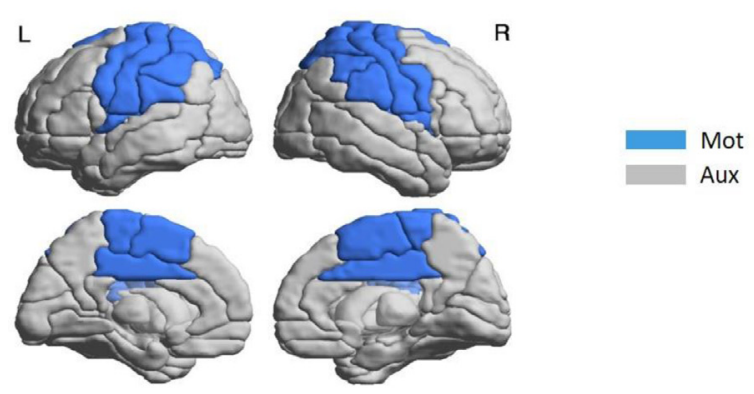

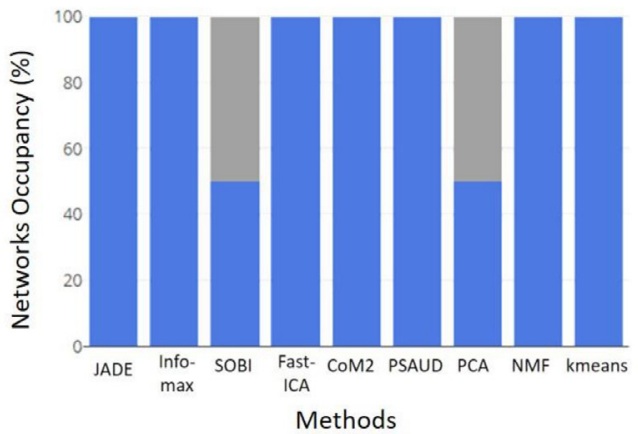

Methods

\section{B. HCP Left-hand Motor Task}
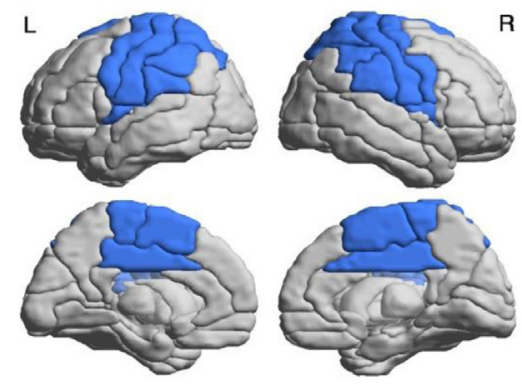

Mot

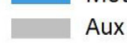

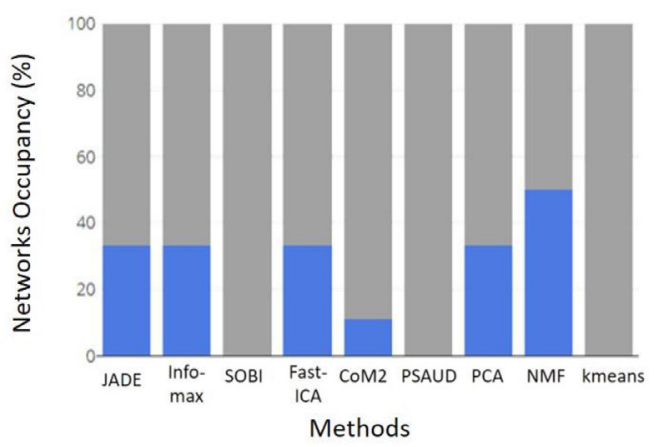

\section{Working Memory Task}

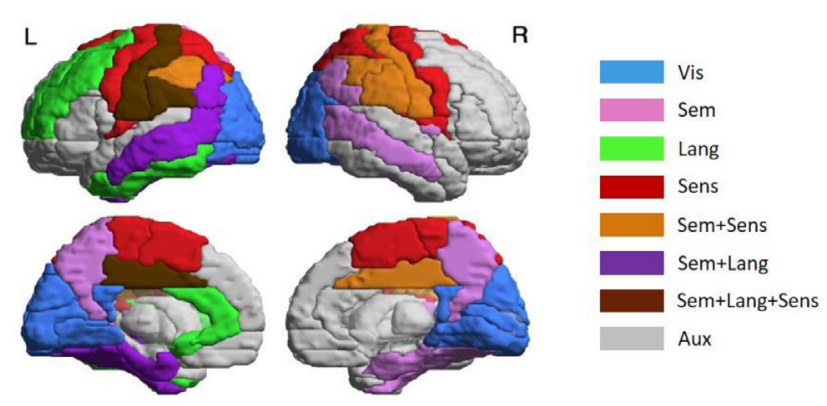

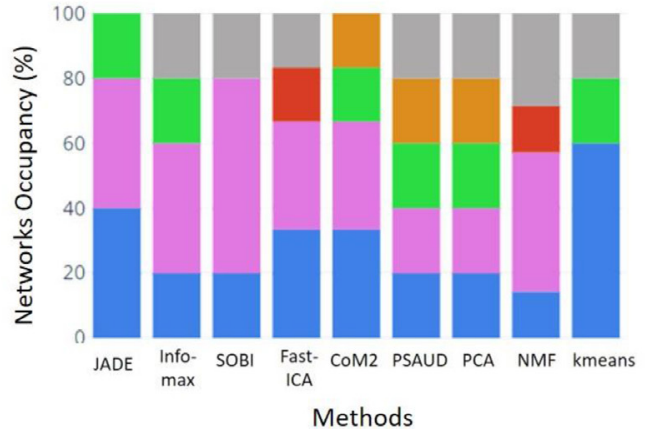

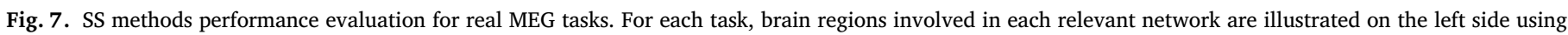

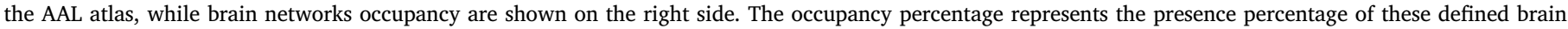

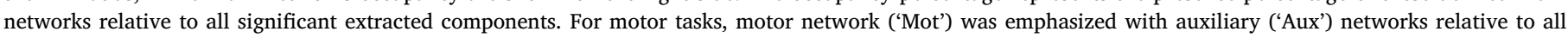

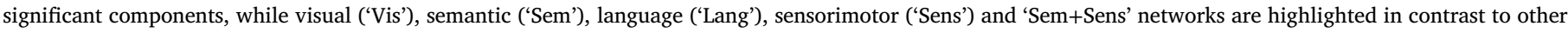

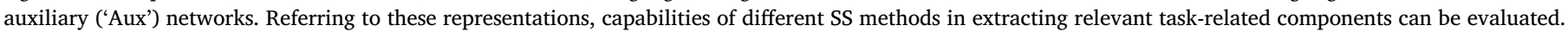

temporal distributions of selected subjects' components in both cases are shown in Fig. 8. Results of the remaining components for these 2 subjects' examples are shown in Supplementary Fig. S11.

As a result, two parameters were collected and represented in Fig. 8.B and 8.C respectively. Group-subject similarity percentage was calculated as the number of subjects that gives a motor component similar to the group-level result relative to the total number of subjects $(\mathrm{N}=15)$. Fig. 8.B illustrates this parameter for all SS methods. We can see that JADE was able to extract a task-related component from 8 out of 15 subjects (53.33\%), InfoMax and PSAUD from 7 subjects (46.67\%), SOBI and CoM2 from 6 (40\%), FastICA from 5 (33\%), PCA and NMF from $4(26.67 \%)$ and Kmeans from $3(20 \%)$. The Fig. 8.C shows the distributions of $\mathrm{AD}$ and $\mathrm{CS}$ values of selected components from each subject over all SS methods. Methods with higher subject-group similarity percentage have lower median values of $\mathrm{AD}$ and higher median values of CS. In addition, we can notice from $\mathrm{AD}$ and $\mathrm{CS}$ median values that similarity in space was much easier to be satisfied than temporal similarity for most SS methods. Interquartile range values show the existence of inter-subject variability results. However, some methods showed higher interquartile range of $\mathrm{AD}$ values (CoM2 and PCA), or CS values (JADE, CoM2 and NMF) relative to other methods.

\subsection{The optimal number of subjects of each SS method}

Then, the same procedure was applied with increasing the number of subjects from one subject (single-subject) to 14 subjects. AD and CS are 


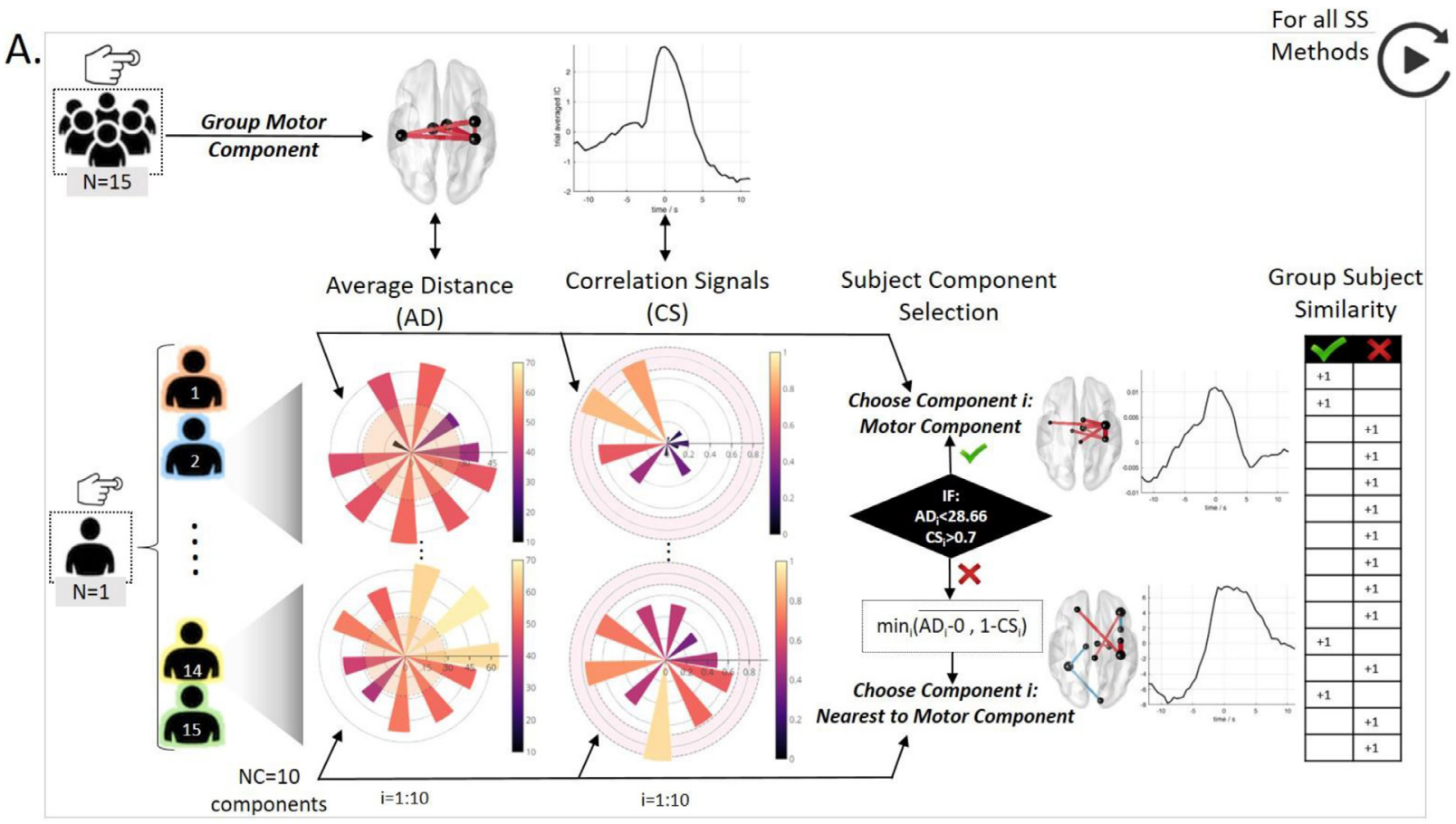

B.

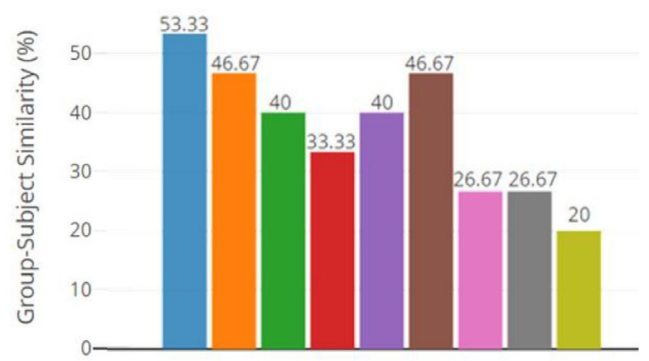

Methods

D.

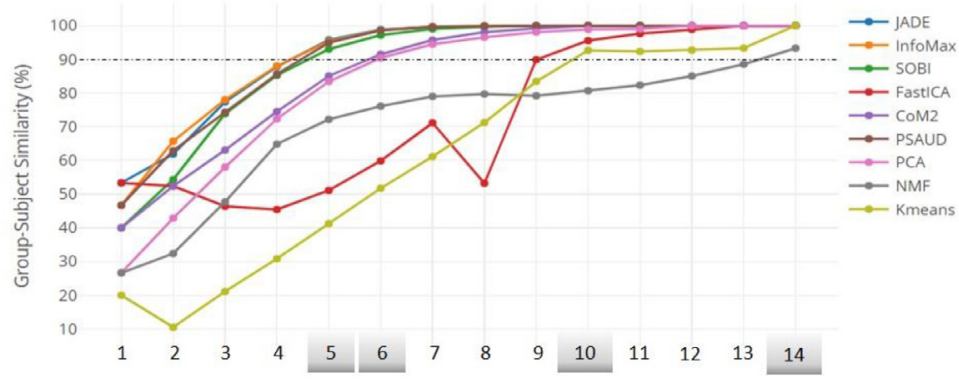

is igs
C.
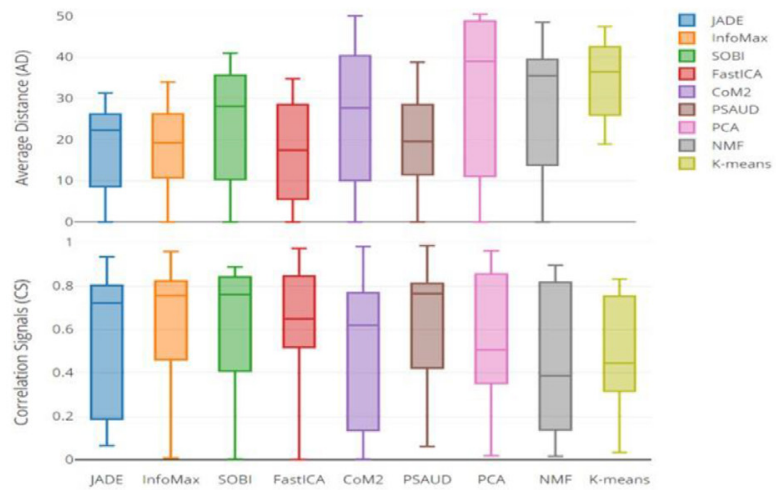

Methods

E.

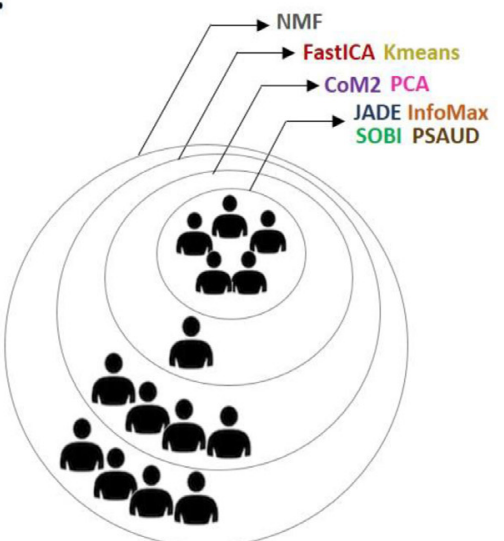

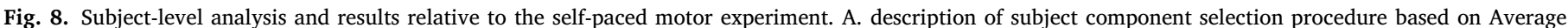

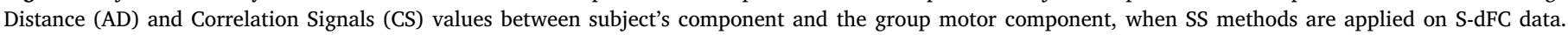

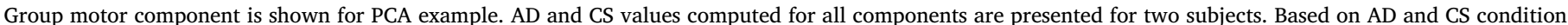

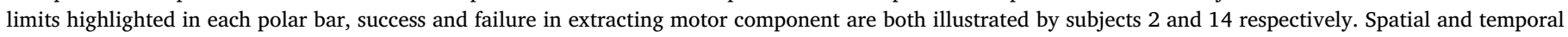

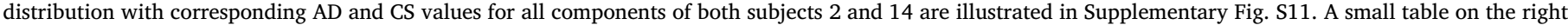

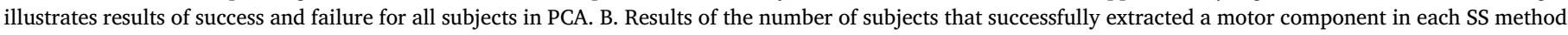

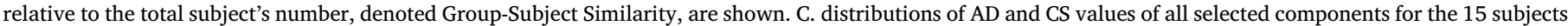

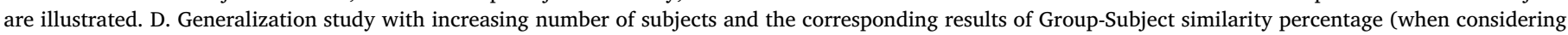

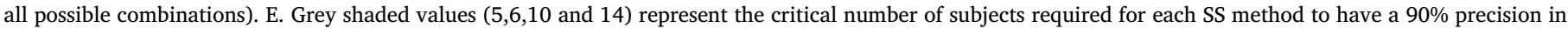
extracting the task related component. 
computed for all possible combinations. The number of possible combinations is calculated. For example, 7-subjects analysis requires 6435 combinations, hence 6435 values of $\mathrm{AD}$ and CS. For each number of subjects, we calculated subject to group similarity as the ratio between number of combinations that succeeded in extracting a motor component relative to the total number of possible combinations. Results are illustrated in Fig. 8.E, F. As expected, the percentage similarity increases with increasing number of subjects for all SS methods. Fluctuations in similarity results are observed in some methods (as FastICA, NMF and Kmeans) due to the non-consistency characteristic of these methods (as previously proved). Some methods required a smaller number of subjects for data analysis to provide satisfactory results (motor component at button press time in our case) than others. For example, the four ICA versions (JADE, InfoMax, SOBI and PSAUD) required 5 subjects in order to attain a minimum similarity level of $90 \%$ between subject and group level results. CoM2 and PCA required 6 subjects, while much more subjects were needed for others (10 subjects for FastICA and Kmeans and 14 for NMF) as showed in Fig. 8.E. Overall, ICA methods and specially those based on the high order statistics (such as JADE) outperform other methods in extracting networks at the subject-level.

\section{Discussion}

In this study, we have evaluated the robustness of the most popular SS methods applied to extract the main brain networks fluctuating during time in order to help researchers make a rational choice (if any) among the multitude of available methods. Specifically, nine algorithms have been compared using simulated data (see supplementary materials) and three independent MEG datasets $(\mathrm{N}=95)$ recorded during motor and memory tasks. The discrepancy in the datasets size and behavioral tasks performed allows testing SS methods performance on different scenarios. As the evoked responses (analyzed here) last for hundreds of milliseconds, we conducted our comparative analysis based on MEG datasets to benefit from the excellent temporal resolution of this technique. However, the same pipeline study can be applied in task-related fMRI context.

Overall, our results show variability between the evaluated SS methods and even between ICA subtypes. The performance of these methods depends on the nature of the task (simple vs complex, slow vs fast time scale tasks). In a simple and relatively slow time scale task (as self-paced button press task), all methods succeeded in tracking spatially and temporally the dynamic brain activity. However, when it comes to much faster task (HCP motor task) or more complex task (Working Memory), some methods (SOBI and Kmeans for instance) showed lower performance in extracting relevant brain networks (as defined by our masks). Results relative to each task will be discussed later in details.

First, the quantitative comparison performed on simulated dynamic networks showed that all SS methods have successfully separated functional networks based on their connectivity time courses. However, spatial and temporal similarities in SOBI were significantly lower than other SS methods, especially for the fourth simulated state (P4) and the second state (P2) as shown in Supplementary Fig. S3, which involves more complex spatiotemporal activity than other states. As expected, FastICA, NMF and Kmeans methods were proved inconsistent with multiple runs. This is caused by the nature of these algorithms that is based on random input initialisations until solution convergence. The noise effect on the results obtained was also tested and showed an increased performance for all methods with higher SNR value with slower convergence to the optimal accuracy for some methods (SOBI, NMF and Kmeans) relative to others. Regarding computation time, CoM2, PCA and PSAUD were the fastest whereas InfoMax and JADE were the slowest. Still, the executed time of these algorithms is sensible to dataset's features (size, complexity, type...). Other metrics such as the number of floating-point operations (FLOPs) required for the algorithm completion could be also tested. We also suggest for future studies to explore other data simulation approaches that build the desired ground-truth brain states based on more realistic modeling (using Neural Mass for instance), however, this may introduce the effect of other parameters in the comparison (forward problem, inverse solution...). Besides simulation approach, some studies attempt to consider fMRI data as a ground truth to quantify and compare SS methods performance in the context of M/EEG studies (Colclough et al., 2016; J et al., 2020).

The method's performance were evaluated on three real MEG datasets already published and tested by previous studies (Casorso et al., 2019; O'Neill et al., 2017; Tewarie et al., 2019a; Vidaurre et al., 2018a; Zhu et al., 2020). According to self-paced motor task, results showed that all SS methods have successfully extracted one or more significant network that involve strong connectivity between sensorimotor regions ('Mot'). For HCP data, a similar sensorimotor network was revealed among significant components in all SS methods except for SOBI, PSAUD and Kmeans. Integrated regions in this network mainly include nodes from central and parietal gyrus. The sensorimotor network is strongly coherent with the task (Melnik et al., 2017; Yousry, 1997) since it requires both movement (through button press or hand movement) and tactile response (as the subject will feel the button or fingers tape). The effect of right-handedness of all participants of self-paced dataset is also revealed by the presence stronger implication of sensorimotor nodes from the right cortex relative to the left one as revealed by the sphere sizes and connections in Fig. 3. It is noteworthy to mention the existence of a network that highlighted significant connections in the visual lobe in JADE for self-paced button press task and most SS methods for HCP task. This network was previously noticed by Oneill et al. studying the same button press task (O'Neill et al., 2017). This can be interpreted as a cross modal synchronization between visual and sensorimotor cortex as previously studied (Bauer et al., 2020). Regarding temporal evolution, it is clear that all networks modulate significantly with the exact button press time for self-paced task. Differently, the temporal variation related to HCP motor task takes an oscillatory shape, which was also reported by other studies dealing with the same dataset (Vidaurre et al., 2018a; Zhu et al., 2020). A possible reason for this activity was suggested by (Vidaurre et al., 2018a) considering a leakage effect of temporal activity of previous button press into the next trial due to the fast successive trials. In both tasks, there exists auxiliary 'Aux' networks that significantly modulated with the task but not directly related to the motor cortex activity. The occupancy percentage of 'Aux' networks increases for all SS methods in HCP results mainly in CoM2 and Kmeans. The presence of these networks can be related either to the robustness/sensitivity of the SS method relative to spurious networks or to the reliability of the techniques used for selection of optimal number of components (DIFFIT) or significant components (null distribution) that will be further discussed later.

In order to evaluate the spatial and temporal accuracy of SS methods at higher levels of complexity, we tested the methods on Sternberg working memory task. All SS methods detected visual network, which is consistent with the presentation of visual stimuli at two different times. Regions in the primary visual lobe related to stimulus visualisation (Grill-Spector et al., 1998) and lateral occipital cortex responsible for object/shape recognition (Corbetta et al., 1991; Grill-Spector et al., 2001; Kourtzi and Kanwisher, 2001) were present in these networks. The button press response is reflected by sensorimotor connections consistently with previous working memory studies (Metzak et al., 2011; Yamashita et al., 2015) only by using CoM2, PSAUD and PCA methods. In order to process and maintain observed stimuli as a way to memorize them, a higher level of cognition is illustrated by a 'semantic network', which mainly encompasses bilateral parietal and temporal areas activation in all SS methods, except for Kmeans. This is coherent with previous studies that demonstrate the evident role of parietal cortex as a workspace for sensory and perceptual processing in working memory framework (Chai et al., 2018) through angular (Frackowiak, 1992; Vandenberghe et al., 1996), precuneus (Cavanna and Trimble, 2006), and hippocampal (Baddeley et al., 2011) areas. Bilateral inferior temporal regions also play important role in semantic processing 
(Nestor et al., 2006; Vigneau et al., 2006). Fusiform gyri, strongly modulated in our results, has also shown a particular concern in this context(Mion et al., 2010). The detection of the 'language' network by JADE, InfoMax, CoM2, PSAUD, PCA and Kmeans methods, was compatible with previous findings (Brookes et al., 2011b; O'Neill et al., 2017). Temporal and parietal lobes were remarkably activated by these methods, mainly the parahippocampal and supramarginal gyri respectively. These regions are critical in memory encoding and retrieval and semantic cognition (Axmacher et al., 2008; Caminiti et al., 2015; Demb et al., 1995; Derrfuss et al., 2004; Deschamps et al., 2014; Vigneau et al., 2006). In a similar (abstract shape based) working memory task, the interpretation of this network was related to a verbalisation naming strategy employed by participants as a way to aid in memory encoding (Caminiti et al., 2015; O'Neill et al., 2017). Therefore, this network's activation may be possible with the task as it modulates strongly with the probe presentation and response time.

It is important to point here that the resultant networks were denoted objectively in this study using a quantification approach. For a better interpretation of the functional significance of results, we built template brain masks, referring to the literature (see table S1 in supplementary materials), from AAL cortical regions. These masks are used for seeding our networks of interest: 'Mot' in motor tasks, 'Vis'/'Sem'/'Lang'/'Sens' in working memory task. Networks were then classified based on their activated nodes strength relative to each mask. In addition, the used masks have distinct spatial distribution, with some shared regions mainly between 'Sem' and 'Sens' networks. However, when we aim to deeply investigate task-related sub-networks, the 'mask' technique seems to have much more complexity related to the specificity of the integrated brain regions and the precision of an accurate threshold in order to appropriately classify networks results.

Although we performed our study on cognitive tasks, it is a topic of great interest to apply this methodology pipeline on resting-state experiments since many studies have shown the dynamic reconfiguration of the brain during rest as well (Kabbara et al., 2017; Liégeois et al., 2019).

Regarding methodological considerations, first, the optimal number of components to be derived was still a challenging question for all SS methods rather than a direct limitation of our algorithms. In this study, we applied two well-known algorithms (DIFFIT and elbow criterion) for a range of number of components to automatically select the optimal number of components relative to each SS method. The evaluated range of NC values was upper limited by 10 components in order to avoid spurious networks. Moreover, we tried to fix the number of components to 10 for all SS methods in the self-paced motor task at the group-level, as already set in a previous work (O'Neill et al., 2017) that used FastICA algorithm and little difference was observed for the overall results.

Second, we should point that not all these components are necessarily essential, especially in the case of simple tasks as motor tasks. To this end, we followed the approach of the null distribution based on sign flipping algorithm (Hunt et al., 2012; O'Neill et al., 2017; Winkler et al., 2014; Zhu et al., 2020) to select only components whose temporal dynamics significantly modulate with the task. In this way, we ensure that brain dynamics relative to the studied behavioural tasks can be summarized and described by the retained components through an automatic way that allows us to objectively compare the SS methods performance. In addition, the fact that this technique is a purely data-driven procedure that does not require any prior hypothesis or conditions manipulation makes it likely adapted to the specific examined dataset. Two points regarding significant components selection are important to mention here. First, in the applied null distribution, networks were defined to be significant if they fell outside the null distribution in either positive or negative sides because they reflect trial-onset-locked that either increases or decreases in connectivity across subjects (as amplitude envelope correlation was adopted). Second, it should be noted that components significance was evaluated relative to the specific task duration. For example, temporal duration of the entire analysis for self-paced and working memory tasks can include dynamics that should be excluded from the analysis. In this context, we limited our significance interpretation/assessment in the interval of $[-2 ;+2 \mathrm{sec}]$ and $[-0.5 ;+0.5 \mathrm{sec}]$ relative to the button press instant in the case of self-paced and HCP motor tasks respectively, and $[-2 ;+16 \mathrm{sec}]$ relative to the visual stimulus presentation for memory task. In addition, few limitations are to be discussed when dealing with this selection. First, it was not convenient to rely on this technique when the number of trials and subjects was either too small or too big. A small number will not allow to build a reliable null distribution while a huge one will have its computational cost regarding all possible subjects' combinations for sign-flipping procedure, as already executed in the HCP analysis. Moreover, there is no consensus about the thresholds/margins that define well a limit level for component's amplitude. For instance, there exists networks whose temporal variation peaks at the limit of null distribution envelope. These are considered to be critical components that may be integrated in the task but considered not to be following the automatic criteria of this null distribution. Future works should therefore investigate more about this methodological approach in the framework of cognitive tasks, in addition to resting state experiments. Also, it is crucial to seek more methods to use or combine with the applied technique in order to have more robust basis for significant components selection. It is noteworthy to report that null distribution-based technique was applied uniformly for all SS methods, thus our main objective of comparison was built on a unified evaluation framework.

Third, we used the same pipeline supported by the previous studies dealing with the same dataset (cortical parcellation, source reconstruction, functional connectivity metric and source leakage correction, frequency bands and sliding window settings) (Kabbara et al., 2019; O’Neill et al., 2017). By applying already tested and validated methodological approaches, we avoid influencing factors on the comparison performed. However, we point out that other methodological solutions could be exploited by other researches using the same pipeline adopted in this work. Regarding cortical parcellation, we chose AAL atlas based on its successful use in previous MEG investigations (O'Neill et al., 2017; Tewarie et al., 2016). This atlas also provides good basis for the orthogonalisation procedure adopted since its number of regions is sufficiently low (78 ROIs) and well separated (Colclough et al., 2015). The beamformer spatial filtering was selected as the inverse problem solution due to its demonstrated efficiency in the measurement of static (Brookes et al., 2011a) and dynamic (Baker et al., 2014) functional connectivity. Functional connectivity between ROIs regions was estimated through Amplitude Envelope Correlation (AEC). This technique has been successful in elucidating electrophysiological networks of functional connectivity (Colclough et al., 2016). Other methods, such as phase couplings can be considered as an alternative way to probe different type of functional connectivity (Lachaux et al., 1999). Sliding window settings (length and step) were selected carefully as a trade-off between temporal resolution and the accuracy of the derived adjacency matrices (length $=6 \mathrm{sec}$, step $=0.5 \mathrm{sec}$ for self-paced and working memory tasks) (O'Neill et al., 2017). However, according to recent works (Fraschini et al., 2016; Liuzzi et al., 2019), it can be seen that metrics (including amplitude envelope correlation) perform poorly for very short state durations when combined with the sliding window approach below few seconds, providing noisy results of low correlation with ground truth in simulations. For this reason, we followed the work of (Tewarie et al., $2019 b$ ) to estimate dynamic functional connectivity by taking sample by sample time series rather than windowed aggregated samples using the Instantaneous Amplitude Correlation (IAC). This high temporal resolution measure of FC has shown great sensitivity to genuine fluctuations in functional connectivity applied in the same context of our study.

It would be interesting to test data-driven windows approach in this context using the recurrence plots of the amplitude envelopes as in (Tewarie et al., 2019b) instead of averaging trials in dataset 2 to reduce heavy dFC matrices. It could be also tested against fixed sliding window approach as for datasets 1 and 3 . 
Concerning frequency bands, it was crucial to preprocess each dataset in its appropriate bandwidth. For example, brain signals in selfpaced and HCP motor tasks were proved to be more active in the beta band, while broader range of frequency bands are integrated in complex cognitive tasks as working memory (O'Neill et al., 2017; Zhu et al., 2020).

\section{Conclusion}

Deciphering of dynamics of electrophysiological brain networks is one of the most important goals in neuroscience. In this paper, we evaluated and compared nine popular source separation (SS) methods to identify dominant networks of connections with corresponding temporal dynamics at group-level as well as subject-level, using simulation and empirical MEG data ( $\mathrm{N}=95$ subjects) recorded during three different tasks: (1) simple button press task, (2) fast finger movement task (HCP) and (3) Sternberg working memory task. Results show close consistency for all SS methods in successfully identifying a transient network of connections linking somatosensory and primary motor regions in the relatively slow and simple button press task. Variability between these methods' performance is revealed in rapid tasks of sub-second timescale (HCP motor task) and in a more complex task (Sternberg). The SOBI and Kmeans algorithms showed the weakest performance among tested methods. CoM2, PSAUD and PCA showed promising results in working memory task, revealing the formation and dissolution of multiple networks that relate to semantic processing, pattern recognition and language as well as vision and movement. At the subject level analysis, ICA methods using high statistical order (JADE, InfoMax, CoM2 and PSAUD) outperform other methods. Our main message is that researchers should be aware to select the appropriate SS methods and other related parameters (epoch length, task complexity and dataset size) when analyzing dynamics of behavioral tasks.

\section{Data availability}

Data supporting the findings of this study are available in the link (https://github.com/judytabbal/dynbrainSS.git). All HCP data are available on https://www.humanconnectome.org/software/hcp-megpipelines. The datasets 1 and 3 are available upon request.

\section{Code availability}

Codes supporting the findings of this study are available in the link (https://github.com/judytabbal/dynbrainSS.git). All analysis codes necessary to produce the results here were performed in MATLAB software, using FieldTrip Toolbox http://www.fieldtriptoolbox.org for data segmentation, filtering and source reconstruction steps, EEGLAB toolbox for some SS methods as JADE, InfoMax and SOBI and other MATLAB implemented functions as detailed and provided in the previous link. A graphical user interface is made freely available allowing other researchers to test the methods on our (or their) simulated and real data.

\section{Credit author statement}

JT, AK, MH and PB contributed to the design and implementation of the research, to the analysis of the results and to the writing of the manuscript. MK and PB were involved in providing funding sources for the study and supervised the work. All authors provided critical feedback and helped shape the study.

\section{Acknowledgement}

This work was financed by the Rennes University and the Institute of Clinical Neuroscience of Rennes (project named EEGCog). The study was also funded by the National Council for Scientific Research (CNRS) in Lebanon. The authors would also like to thank the the Lebanese
University, the Lebanese Association for Scientific Research (LASER) and Campus France, Programme Hubert Curien CEDRE (PROJECT No. 42257YA), for supporting this study.

\section{Supplementary materials}

Supplementary material associated with this article can be found, in the online version, at doi:10.1016/j.neuroimage.2021.117829.

\section{References}

Aggarwal, C.C., Hinneburg, A., Keim, D.A., 2001. On the surprising behavior of distance metrics in high dimensional space. In: Van den Bussche, J., Vianu, V. (Eds.), Database Theory - ICDT 2001, Lecture Notes in Computer Science. Springer, Berlin, Heidelberg, pp. 420-434. doi:10.1007/3-540-44503-X_27.

Allen, E.A., Damaraju, E., Plis, S.M., Erhardt, E.B., Eichele, T., Calhoun, V.D., 2014. Tracking whole-brain connectivity dynamics in the resting state. Cereb Cortex 24, 663-676. doi:10.1093/cercor/bhs352.

Axmacher, N., Schmitz, D.P., Wagner, T., Elger, C.E., Fell, J., 2008. Interactions between medial temporal lobe, prefrontal cortex, and inferior temporal regions during visual working memory: a combined intracranial eeg and functional magnetic resonance imaging study. J. Neurosci. 28, 7304-7312. doi:10.1523/JNEUROSCI.1778-08.2008.

Baddeley, A., Jarrold, C., Vargha-Khadem, F., 2011. Working memory and the hippocampus. J. Cogn. Neurosci. 23, 3855-3861. doi:10.1162/jocn a 00066.

Baker, A.P., Brookes, M.J., Rezek, I.A., Smith, S.M., Behrens, T., Probert Smith, P.J., Woolrich, M., 2014. Fast transient networks in spontaneous human brain activity. eLife 3, e01867. doi:10.7554/eLife.01867.

Bassett, D.S., Sporns, O., 2017. Network neuroscience. Nature Neurosci. 20, 353.

Bauer, A.-K.R., Debener, S., Nobre, A.C., 2020. Synchronisation of neural oscillations and cross-modal Influences. Trends Cognit. Sci. 24, 481-495. doi:10.1016/j.tics.2020.03.003.

Bola, M., Sabel, B.A., 2015. Dynamic reorganization of brain functional networks during cognition. Neuroimage 114, 398-413.

Brookes, M.J., Hale, J.R., Zumer, J.M., Stevenson, C.M., Francis, S.T., Barnes, G.R., Owen, J.P., Morris, P.G., Nagarajan, S.S., 2011a. Measuring functional connectivity using MEG: methodology and comparison with fcMRI. Neuroimage 56, 1082-1104. doi:10.1016/j.neuroimage.2011.02.054.

Brookes, M.J., Tewarie, P.K., Hunt, B.A.E., Robson, S.E., Gascoyne, L.E., Liddle, E.B., Liddle, P.F., Morris, P.G., 2016. A multi-layer network approach to MEG connectivity analysis. NeuroImage 132, 425-438. doi:10.1016/j.neuroimage.2016.02.045.

Brookes, M.J., Vrba, J., Robinson, S.E., Stevenson, C.M., Peters, A.M., Barnes, G.R., Hillebrand, A., Morris, P.G., 2008. Optimising experimental design for MEG beamformer imaging. NeuroImage 39, 1788-1802. doi:10.1016/j.neuroimage.2007.09.050.

Brookes, M.J., Wood, J.R., Stevenson, C.M., Zumer, J.M., White, T.P., Liddle, P.F., Morris, P.G., 2011b. Changes in brain network activity during working memory tasks: a magnetoencephalography study. Neuroimage 55, 1804-1815. doi:10.1016/j.neuroimage.2010.10.074.

Brookes, M.J., Woolrich, M.W., Barnes, G.R., 2012. Measuring functional connectivity in MEG: a multivariate approach insensitive to linear source leakage. Neuroimage 63 , 910-920. doi:10.1016/j.neuroimage.2012.03.048.

Buckner, R.L., Krienen, F.M., Castellanos, A., Diaz, J.C., Yeo, B.T.T., 2011. The organization of the human cerebellum estimated by intrinsic functional connectivity. J Neurophysiol 106, 2322-2345. doi:10.1152/jn.00339.2011.

Bullmore, E., Sporns, O., 2009. Complex brain networks: graph theoretical analysis of structural and functional systems. Nat. Rev. Neurosci. 10, 186-198. doi:10.1038/nrn2575.

Caminiti, S.P., Siri, C., Guidi, L., Antonini, A., Perani, D., 2015. The neural correlates of spatial and object working memory in elderly and Parkinson's disease subjects. Behav. Neurol. 2015, 1-10. doi:10.1155/2015/123636.

Casorso, J., Kong, X., Chi, W., Van De Ville, D., Yeo, B.T.T., Liégeois, R., 2019. Dynamic mode decomposition of resting-state and task fMRI. NeuroImage 194, 42-54. doi:10.1016/j.neuroimage.2019.03.019.

Cavanna, A.E., Trimble, M.R., 2006. The precuneus: a review of its functional anatomy and behavioural correlates. Brain 129, 564-583. doi:10.1093/brain/aw1004.

Chai, L.R., Khambhati, A.N., Ciric, R., Moore, T.M., Gur, R.C., Gur, R.E., Satterthwaite, T.D., Bassett, D.S., 2017. Evolution of brain network dynamics in neurodevelopment. Network Neurosci. 1, 14-30. doi:10.1162/NETN_a_00001.

Chai, W.J., Abd Hamid, A.I., Abdullah, J.M., 2018. Working memory from the psychological and neurosciences perspectives: a review. Front Psychol. 9. doi:10.3389/fpsyg.2018.00401.

Ciric, R., Nomi, J.S., Uddin, L.Q., Satpute, A.B., 2017. Contextual connectivity: a framework for understanding the intrinsic dynamic architecture of large-scale functional brain networks. Sci. Reports 7, 1-16. doi:10.1038/s41598-017-06866-w.

Colclough, G.L., Brookes, M.J., Smith, S.M., Woolrich, M.W., 2015. A symmetric multivariate leakage correction for MEG connectomes. Neuroimage 117, 439-448. doi:10.1016/j.neuroimage.2015.03.071.

Colclough, G.L., Woolrich, M.W., Tewarie, P.K., Brookes, M.J., Quinn, A.J., Smith, S.M., 2016. How reliable are MEG resting-state connectivity metrics? Neuroimage 138, 284-293. doi:10.1016/j.neuroimage.2016.05.070.

Corbetta, M., Miezin, F.M., Dobmeyer, S., Shulman, G.L., Petersen, S.E., 1991. Selective and divided attention during visual discriminations of shape, color, and speed: functional anatomy by positron emission tomography. J. Neurosci. 11, 2383-2402.

Demb, J.B., Desmond, J.E., Wagner, A.D., Vaidya, C.J., Glover, G.H., Gabrieli, J.D., 1995 Semantic encoding and retrieval in the left inferior prefrontal cortex: a functional 
MRI study of task difficulty and process specificity. J. Neurosci. 15, 5870-5878. doi:10.1523/JNEUROSCI.15-09-05870.1995.

Derrfuss, J., Brass, M., Yves von Cramon, D., 2004. Cognitive control in the posterior frontolateral cortex: evidence from common activations in task coordination, interference control, and working memory. NeuroImage 23, 604-612. doi:10.1016/j.neuroimage.2004.06.007

Deschamps, I., Baum, S.R., Gracco, V.L., 2014. On the role of the supramarginal gyrus in phonological processing and verbal working memory: evidence from rTMS studies. Neuropsychologia 53, 39-46. doi:10.1016/j.neuropsychologia.2013.10.015.

Ding, X., Lee, J.-H., Lee, S.-W., 2013. Performance evaluation of nonnegative matrix factorization algorithms to estimate task-related neuronal activities from fMRI data. Magn. Reson. Imaging 31, 466-476. doi:10.1016/j.mri.2012.10.003.

Du, Y., Pearlson, G.D., Yu, Q., He, H., Lin, D., Sui, J., Wu, L., Calhoun, V.D., 2016. Interaction among subsystems within default mode network diminished in schizophrenia patients: a dynamic connectivity approach. Schizophr. Res. 170, 5565. doi:10.1016/j.schres.2015.11.021.

Fong, A.H.C., Yoo, K., Rosenberg, M.D., Zhang, S., Li, C.-S.R., Scheinost, D., Constable, R.T., Chun, M.M., 2019. Dynamic functional connectivity during task performance and rest predicts individual differences in attention across studies. NeuroImage 188, 14-25. doi:10.1016/j.neuroimage.2018.11.057.

Fontolan, L., Morillon, B., Liegeois-Chauvel, C., Giraud, A.-L., 2014. The contribution of frequency-specific activity to hierarchical information processing in the human auditory cortex. Nat. Commun. 5, 4694. doi:10.1038/ncomms5694.

Frackowiak, R., 1992. The anatomy of phonological and semantic processing in normal subjects. Brain 115, 1753-1768 version 1 - 2 Dec 2008. Brain 671-682.

Fraschini, M., Demuru, M., Crobe, A., Marrosu, F., Stam, C.J., Hillebrand, A., 2016. The effect of epoch length on estimated EEG functional connectivity and brain network organisation. J. Neural Eng. 13, 036015. doi:10.1088/1741-2560/13/3/036015.

Friston, K.J., 1994. Functional and effective connectivity in neuroimaging: A synthesis. Human Brain Mapp. 2, 56-78. doi:10.1002/hbm.460020107.

Golub, G.H., Reinsch, C., 1970. Singular value decomposition and least squares solutions. Numer. Math. 14, 403-420. doi:10.1007/BF02163027.

Gong, G., He, Y., Concha, L., Lebel, C., Gross, D.W., Evans, A.C., Beaulieu, C., 2009. Mapping anatomical connectivity patterns of human cerebral cortex using in vivo diffusion tensor imaging tractography. Cerebral Cortex (New York, N.Y: 1991) 19, 524-536. doi:10.1093/cercor/bhn102

Grill-Spector, K., Kourtzi, Z., Kanwisher, N., 2001. The lateral occipital complex and its role in object recognition. Vision Res. 41, 1409-1422. doi:10.1016/S0042-6989(01)00073-6.

Grill-Spector, K., Kushnir, T., Edelman, S., Itzchak, Y., Malach, R., 1998. Cue-invariant activation in object-related areas of the human occipital lobe. Neuron 21, 191-202. doi:10.1016/S0896-6273(00)80526-7.

Hassan, M., Benquet, P., Biraben, A., Berrou, C., Dufor, O., Wendling, F., 2015. Dynamic reorganization of functional brain networks during picture naming. Cortex 73, 276288. doi:10.1016/j.cortex.2015.08.019.

Hassan, M., Dufor, O., Merlet, I., Berrou, C., Wendling, F., 2014. EEG source connectivity analysis: from dense array recordings to brain networks. PloS one 9, e105041.

Hassan, M., Wendling, F., 2018. Electroencephalography source connectivity: aiming for high resolution of brain networks in time and space. IEEE Signal Process. Mag. 35, 81-96. doi:10.1109/MSP.2017.2777518.

Hillebrand, A., Tewarie, P., van Dellen, E., Yu, M., Carbo, E.W.S., Douw, L., Gouw, A.A., van Straaten, E.C.W., Stam, C.J., 2016. Direction of information flow in large-scale resting-state networks is frequency-dependent. Proc. Nat. Acad. Sci. USA 113, 3867 3872. doi:10.1073/pnas.1515657113

Hipp, J.F., Hawellek, D.J., Corbetta, M., Siegel, M., Engel, A.K., 2012. Large-scale cortical correlation structure of spontaneous oscillatory activity. Nat. Neurosci. 15, 884-890. doi: $10.1038 / \mathrm{nn} .3101$

Hunt, L.T., Kolling, N., Soltani, A., Woolrich, M.W., Rushworth, M.F.S., Behrens, T.E.J., 2012. Mechanisms underlying cortical activity during value-guided choice. Nature Neuroscience 15, 470-476. doi:10.1038/nn.3017.

Iraji, A., Faghiri, A., Lewis, N., Fu, Z., Rachakonda, S., Calhoun, V., 2020. Tools of the Trade: Estimating Time-Varying Connectivity Patterns from fMRI Data (preprint) PsyArXiv doi:10.31234/osf.io/mvqj4.

J, R., H, A., M, F., F, W., M, H., 2020. Exploring the correlation between M/EEG source-space and fMRI networks at rest. Brain Topogr. 33, 151-160. doi:10.1007/s10548-020-00753-w.

Kabbara, A., Falou, W.E., Khalil, M., Wendling, F., Hassan, M., 2017. The dynamic functional core network of the human brain at rest. Scientific Reports 7, 2936.

Kabbara, A., Khalil, M., O’Neill, G., Dujardin, K., El Traboulsi, Y., Wendling, F., Hassan, M., 2019. Detecting modular brain states in rest and task. Network Neurosci. 1-24.

Kabbara, A., Paban, V., Hassan, M., 2020. The Dynamic Modular Fingerprints of the Human Brain at Rest bioRxiv.

Kachenoura, A., Albera, L., Senhadji, L., Comon, P., 2008. ICA: a potential tool for bci systems. IEEE Signal Process. Mag. 25, 57-68. doi:10.1109/MSP.2008.4408442.

Kourtzi, Z., Kanwisher, N., 2001. Representation of perceived object shape by the human lateral occipital complex. Science 293, 1506-1509. doi:10.1126/science.1061133.

Lachaux, J.-P., Rodriguez, E., Martinerie, J., Varela, F.J., 1999. Measuring phase synchrony in brain signals. Human Brain Mapp. 8, 194-208 https://doi.org/10.1002/(SICI)1097-0193(1999)8:4<194::AID-HBM4 > 3.0.CO;2-C.

Lee, D.D., Seung, H.S., 1999. Learning the parts of objects by non-negative matrix factorization. Nature 401, 788-791. doi:10.1038/44565.

Leonardi, N., Richiardi, J., Gschwind, M., Simioni, S., Annoni, J.-M., Schluep, M., Vuilleumier, P., Van De Ville, D., 2013. Principal components of functional connectivity: a new approach to study dynamic brain connectivity during rest. NeuroImage 83, 937950. doi:10.1016/j.neuroimage.2013.07.019.
Liégeois, R., Li, J., Kong, R., Orban, C., Van De Ville, D., Ge, T., Sabuncu, M.R., Yeo, B.T.T., 2019. Resting brain dynamics at different timescales capture distinct aspects of human behavior. Nature Commun. 10, 2317. doi:10.1038/s41467-019-10317-7.

Liu, X., Duyn, J.H., 2013. Time-varying functional network information extracted from brief instances of spontaneous brain activity. PNAS 110, 4392-4397. doi:10.1073/pnas.1216856110.

Liuzzi, L., Quinn, A.J., O'Neill, G.C., Woolrich, M.W., Brookes, M.J., Hillebrand, A., Tewarie, P., 2019. How sensitive are conventional MEG functional connectivity metrics with sliding windows to detect genuine fluctuations in dynamic functional connectivity? Front. Neurosci. 13. doi:10.3389/fnins.2019.00797.

Lloyd, S., 1982. Least squares quantization in PCM. IEEE Trans. Inform. Theory 28, 129 137. doi:10.1109/TIT.1982.1056489.

Melnik, A., Hairston, W.D., Ferris, D.P., König, P., 2017. EEG correlates of sensorimotor processing: independent components involved in sensory and motor processing. Sci. R. 7, 4461. doi:10.1038/s41598-017-04757-8.

Metzak, P., Feredoes, E., Takane, Y., Wang, L., Weinstein, S., Cairo, T., Ngan, E.T.C., Woodward, T.S., 2011. Constrained principal component analysis reveals functionally connected load-dependent networks involved in multiple stages of working memory. Hum. Brain Mapp. 32, 856-871. doi:10.1002/hbm.21072.

Mheich, A., Hassan, M., Khalil, M., Berrou, C., Wendling, F., 2015. A new algorithm for spatiotemporal analysis of brain functional connectivity. J. Neurosci. Methods 242, 77-81. doi:10.1016/j.jneumeth.2015.01.002.

Mheich, A., Hassan, M., Khalil, M., Gripon, V., Dufor, O., Wendling, F., 2018. SimiNet: a novel method for quantifying brain network similarity. IEEE Trans. Pattern Anal. Mach. Intell. 40, 2238-2249. doi:10.1109/TPAMI.2017.2750160.

Miller, R.L., Yaesoubi, M., Turner, J.A., Mathalon, D., Preda, A., Pearlson, G., Adali, T., Calhoun, V.D., 2016. Higher dimensional meta-state analysis reveals reduced resting fMRI connectivity dynamism in schizophrenia patients. PLOS ONE 11, e0149849. doi:10.1371/journal.pone.0149849.

Mion, M., Patterson, K., Acosta-Cabronero, J., Pengas, G., Izquierdo-Garcia, D., Hong, Y.T. Fryer, T.D., Williams, G.B., Hodges, J.R., Nestor, P.J., 2010. What the left and right anterior fusiform gyri tell us about semantic memory. Brain 133, 3256-3268. doi:10.1093/brain/awq272

Negrón-Oyarzo, I., Espinosa, N., Aguilar-Rivera, M., Fuenzalida, M., Aboitiz, F., Fuentealba, P., 2018. Coordinated prefrontal-hippocampal activity and navigation strategy-related prefrontal firing during spatial memory formation. Proc. Natl. Acad. Sci. U.S.A. 115, 7123-7128. doi:10.1073/pnas.1720117115.

Nestor, P.J., Fryer, T.D., Hodges, J.R., 2006. Declarative memory impairments in Alzheimer's disease and semantic dementia. Neuroimage 30, 1010-1020. doi:10.1016/j.neuroimage.2005.10.008.

O’Neill, G.C., Bauer, M., Woolrich, M.W., Morris, P.G., Barnes, G.R., Brookes, M.J., 2015. Dynamic recruitment of resting state sub-networks. NeuroImage 115, 85-95. doi:10.1016/j.neuroimage.2015.04.030.

O'Neill, G.C., Tewarie, P.K., Colclough, G.L., Gascoyne, L.E., Hunt, B.A.E., Morris, P.G., Woolrich, M.W., Brookes, M.J., 2017. Measurement of dynamic task related functional networks using MEG. NeuroImage 146, 667-678. doi:10.1016/j.neuroimage.2016.08.061.

Pomper, U., Keil, J., Foxe, J.J., Senkowski, D., 2015. Intersensory selective attention and temporal orienting operate in parallel and are instantiated in spatially distinct sensory and motor cortices: human Intersensory and Temporal Attention. Hum. Brain Mapp. 36, 3246-3259. doi:10.1002/hbm.22845.

Robinson, S., 1999. Functional neuroimaging by synthetic aperture magnetometry (SAM). Recent Advances in Biomagnetism.

Rouhinen, S., Siebenhühner, F., Palva, J.M., Palva, S., 2020. Spectral and anatomical patterns of large-scale synchronization predict human attentional capacity. Cereb. Cortex doi:10.1093/cercor/bhaa110.

Sahonero-Alvarez, G., Calderon, H., 2017. A comparison of SOBI, FastICA. JADE Infomax Algorithms 6.

Sarvas, J., 1987. Basic mathematical and electromagnetic concepts of the biomagnetic inverse problem. Phys. Med. Biol. 32, 11-22. doi:10.1088/0031-9155/32/1/004

Sekihara, K., Nagarajan, S.S., 2008. Adaptive spatial filters for electromagnetic brain imaging. Series in Biomedical Engineering. Springer-Verlag, Berlin Heidelberg doi:10.1007/978-3-540-79370-0.

Smith, S.M., Jenkinson, M., Woolrich, M.W., Beckmann, C.F., Behrens, T.E.J., JohansenBerg, H., Bannister, P.R., De Luca, M., Drobnjak, I., Flitney, D.E., Niazy, R.K., Saunders, J., Vickers, J., Zhang, Y., De Stefano, N., Brady, J.M., Matthews, P.M., 2004 Advances in functional and structural MR image analysis and implementation as FSL. NeuroImage 23 (Suppl 1), S208-S219. doi:10.1016/j.neuroimage.2004.07.051.

Tewarie, P., Bright, M.G., Hillebrand, A., Robson, S.E., Gascoyne, L.E., Morris, P.G., Meier, J., Van Mieghem, P., Brookes, M.J., 2016. Predicting haemodynamic networks using electrophysiology: the role of non-linear and cross-frequency interactions. Neuroimage 130, 273-292. doi:10.1016/j.neuroimage.2016.01.053.

Tewarie, P., Hunt, B.A.E., O’Neill, G.C., Byrne, A., Aquino, K., Bauer, M., Mullinger, K.J., Coombes, S., Brookes, M.J., 2019a. Relationships between neuronal oscillatory amplitude and dynamic functional connectivity. Cereb. Cortex 29, 2668-2681. doi:10.1093/cercor/bhy136.

Tewarie, P., Liuzzi, L., O'Neill, G.C., Quinn, A.J., Griffa, A., Woolrich, M.W., Stam, C.J., Hillebrand, A., Brookes, M.J., 2019b. Tracking dynamic brain networks using high temporal resolution MEG measures of functional connectivity. NeuroImage 200, 3850. doi:10.1016/j.neuroimage.2019.06.006.

Thomas Yeo, B.T., Krienen, F.M., Sepulcre, J., Sabuncu, M.R., Lashkari, D. Hollinshead, M., Roffman, J.L., Smoller, J.W., Zöllei, L., Polimeni, J.R., Fischl, B., Liu, H., Buckner, R.L., 2011. The organization of the human cerebral cortex estimated by intrinsic functional connectivity. J. Neurophysiol. 106, 1125-1165. doi:10.1152/jn.00338.2011 
Timmerman, M.E., Kiers, H.A., 2000. Three-mode principal components analysis: choosing the numbers of components and sensitivity to local optima. Br. J. Math. Stat. Psychol. 53 (Pt 1), 1-16. doi:10.1348/000711000159132.

Van Essen, D.C., Ugurbil, K., Auerbach, E., Barch, D., Behrens, T.E.J., Bucholz, R., Chang, A., Chen, L., Corbetta, M., Curtiss, S.W., Della Penna, S., Feinberg, D., Glasser, M.F., Harel, N., Heath, A.C., Larson-Prior, L., Marcus, D., Michalareas, G., Moeller, S., Oostenveld, R., Petersen, S.E., Prior, F., Schlaggar, B.L., Smith, S.M., Snyder, A.Z., Xu, J., Yacoub, E.WU-Minn HCP Consortium, 2012. The human connectome project: a data acquisition perspective. Neuroimage 62, 2222-2231. doi:10.1016/j.neuroimage.2012.02.018

Vandenberghe, R., Price, C., Wise, R., Josephs, O., Frackowiak, R.S.J., 1996. Functional anatomy of a common semantic system for words and pictures. Nature 383, 254-256. doi:10.1038/383254a0.

Vidaurre, D., Abeysuriya, R., Becker, R., Quinn, A.J., Alfaro-Almagro, F., Smith, S.M., Woolrich, M.W., 2018a. Discovering dynamic brain networks from big data in rest and task. NeuroImage, Brain Connect. Dynamics 180, 646-656. doi:10.1016/j.neuroimage.2017.06.077.

Vidaurre, D., Hunt, L.T., Quinn, A.J., Hunt, B.A.E., Brookes, M.J., Nobre, A.C., Woolrich, M.W., 2018b. Spontaneous cortical activity transiently organises into frequency specific phase-coupling networks. Nature Commun. 9, 2987. doi:10.1038/s41467-018-05316-Z

Vidaurre, D., Quinn, A.J., Baker, A.P., Dupret, D., Tejero-Cantero, A., Woolrich, M.W., 2016. Spectrally resolved fast transient brain states in electrophysiological data. NeuroImage 126, 81-95. doi:10.1016/j.neuroimage.2015.11.047.

Vigneau, M., Beaucousin, V., Hervé, P.Y., Duffau, H., Crivello, F., Houdé, O., Mazoyer, B., Tzourio-Mazoyer, N., 2006. Meta-analyzing left hemisphere language ar- eas: phonology, semantics, and sentence processing. Neuroimage 30, 1414-1432. doi:10.1016/j.neuroimage.2005.11.002.

Wang, D., Zhu, Y., Ristaniemi, T., Cong, F., 2018. Extracting multi-mode ERP features using fifth-order nonnegative tensor decomposition. J. Neurosci. Methods 308, 240 247. doi:10.1016/j.jneumeth.2018.07.020.

Wilkins, K.B., Yao, J., 2020. Coordination of multiple joints increases bilateral connectivity with ipsilateral sensorimotor cortices. Neuroimage 207, 116344 doi:10.1016/j.neuroimage.2019.116344.

Winkler, A.M., Ridgway, G.R., Webster, M.A., Smith, S.M., Nichols, T.E., 2014 Permutation inference for the general linear model. Neuroimage 92, 381-397. doi:10.1016/j.neuroimage.2014.01.060,

Yaesoubi, M., Miller, R.L., Calhoun, V.D., 2015. Mutually temporally independent connectivity patterns: A new framework to study the dynamics of brain connectivity at rest with application to explain group difference based on gender. NeuroImage 107, 85-94. doi:10.1016/j.neuroimage.2014.11.054.

Yamashita, M., Kawato, M., Imamizu, H., 2015. Predicting learning plateau of working memory from whole-brain intrinsic network connectivity patterns. Sci. Rep. 5, 1-8. doi:10.1038/srep07622.

Yousry, T., 1997. Localization of the motor hand area to a knob on the precentral gyrus. A new landmark. Brain 120, 141-157. doi:10.1093/brain/120.1.141.

Zhu, Y., Liu, J., Ye, C., Mathiak, K., Astikainen, P., Ristaniemi, T., Cong, F., 2020. Discovering dynamic task-modulated functional networks with specific spectral modes using MEG. NeuroImage 218, 116924. doi:10.1016/j.neuroimage.2020.116924. 